\title{
Location-Aided Uplink Transmission for User- Centric Cell-Free Massive MIMO Systems: A Fairness Priority Perspective
}

\section{Chen Wei}

Army Engineering University of PLA

Kui Xu ( $D$ Igdxxukui@sina.com)

College of Communications Engineering, PLA University of Science and Technology https://orcid.org/0000-0001-8533-2255

\section{Zhexian Shen}

Army Engineering University of PLA

\section{Xiaochen Xia}

Army Engineering University of PLA

Wei Xie

Army Engineering University of PLA

Chunguo Li

Southeast University

\section{Research Article}

Keywords: user-centric cell-free massive MIMO, pilot assignment, location-aided distributed uplink combining, low-complexity, fairness

Posted Date: January 14th, 2022

DOI: https://doi.org/10.21203/rs.3.rs-1202385/v1

License: (c) (1) This work is licensed under a Creative Commons Attribution 4.0 International License. Read Full License 


\title{
Location-Aided Uplink Transmission for User-Centric Cell-Free Massive MIMO Systems: A Fairness Priority Perspective
}

\author{
Chen Wei, Kui Xu* ${ }^{*}$ Zhexian Shen, Xiaochen Xia, Wei Xie and Chunguo Li
}

\author{
${ }^{*}$ Correspondence: \\ Igdxxukui@sina.com \\ College of Communications \\ Engineering, Army Engineering \\ University of PLA, Nanjing, China \\ Full list of author information is \\ available at the end of the article
}

\begin{abstract}
In this paper, we investigate the uplink transmission for user-centric cell-free massive multiple-input multiple-output (MIMO) systems. The

largest-large-scale-fading-based access point (AP) selection method is adopted to achieve a user-centric operation. Under this user-centric framework, we propose a novel inter-cluster interference-based (IC-IB) pilot assignment scheme to alleviate pilot contamination. Considering the local characteristics of channel estimates and statistics, we propose a location-aided distributed uplink combining scheme based on a novel proposed metric representing inter-user interference to balance the relationship among the spectral efficiency (SE), user equipment (UE) fairness and complexity, in which the normalized local partial minimum mean-squared error (LP-MMSE) combining is adopted for some APs, while the normalized maximum ratio (MR) combining is adopted for the remaining APs. A new closed-form SE expression using the normalized MR combining is derived and a novel metric to indicate the UE fairness is also proposed. Moreover, the max-min fairness (MMF) power control algorithm is utilized to further ensure uniformly good service to the UEs. Simulation results demonstrate that the channel estimation accuracy of our proposed IC-IB pilot assignment scheme outperforms that of the conventional pilot assignment schemes. Furthermore, although the proposed location-aided uplink combining scheme is not always the best in terms of the per-UE SE, it can provide the more fairness among UEs and can achieve a good trade-off between the average SE and computational complexity.
\end{abstract}

Keywords: user-centric cell-free massive MIMO; pilot assignment; location-aided distributed uplink combining; low-complexity; fairness

\section{Introduction}

Cell-free massive multiple-input multiple-output (MIMO) is a promising technique for beyond-5G and $6 \mathrm{G}$ networks due to its ability to provide a high spectral efficiency (SE) and tremendous macro-diversity with simple signal processing [1-3]. In cellfree massive MIMO systems, lots of access points (APs) jointly provide services to all user equipments (UEs) in the same time-frequency resource by utilizing the centralized coordination of the central processing unit (CPU), which is connected to all APs via fronthaul links [4].

The early studies on cell-free massive MIMO assumed that all APs serve all UEs in the network [5,6]. Unfortunately, this assumption may lead to network-wide fronthaul signaling and data sharing, resulting in huge computational complexity, which is impractical for actual large networks [7]. To overcome this limitation, a 
user-centric framework has been introduced into cell-free massive MIMO; the main idea of this framework is that each UE is served by a subset of APs with the best channel conditions to improve the service efficiency of the system [8-10].

In cell-free massive MIMO, there are two data transmission implementations consisting of the centralized and distributed signal processing, which are characterized by different degrees of cooperation among the APs [11]. Take the uplink as an example, in distributed signal processing, the channel estimation and receive combining are done at the APs, and data detection is done at the CPU. While in centralized processing, all operations mentioned above are done at the CPU, which leads to increased fronthaul load and deployment cost compared with the former. Nowadays, the distributed signal processing has been widely investigated due to its advantages of saving fronthaul overhead and flexible deployment [7, 11-13]. In [7], a new framework for scalable cell-free massive MIMO that exploiting dynamic cooperation cluster (DCC) concept was proposed, and the uplink/downlink SE performance with maximum-ratio (MR) or local partial minimum mean-squared error (LP-MMSE) combining/precoding were studied respectively. Based on the same DCC framework, the authors in [13] proposed new scalable forms of regularized zero-forcing (RZF) combining and signal-to-leakage-and-noise ratio (SLNR) precoding for cellfree massive MIMO.

There methods mentioned above are fully distributed and widely used in cell-free massive MIMO systems. However, the shortcomings of each scheme also obvious. For example, the SE performance of MR combining/precoding is poor, although it has low complexity and good fairness among UEs. On the contrary, the LP-MMSE/RZF combining or LP-MMSE/SLNR precoding outperform the standard MR method in terms of SE performance at the cost of increased computational complexity. Meanwhile, the UE fairness of these methods cannot be ensured effectively $[7,11]$. Based on these observations, a natural thought is the following: can we explore a novel transmission scheme that can make an effective compromise between SE, UE fairness and complexity to improve comprehensive system performance?

\subsection{Motivation}

Driven by the continuously increasing demands for high system throughput, low latency and improved fairness, location-aware communication for beyond-5G and $6 \mathrm{G}$ networks has attracted lots of research interest since location information can be leveraged in wireless network design and optimization to complement existing technological developments $[14,15]$. In massive MIMO systems, especially in cellfree massive MIMO, user location is closely related to its signal spatial structure. The reason is that under the cell-free distributed framework, multiple APs provide services to UEs at the same time-frequency, and the signals from each AP should be coherently converged at one point.

In fact, lots of papers have focused on improving system performance or simplify system design for cellular massive MIMO systems by using location information obtained by means of global positioning system (GPS) or wireless positioning technologies, which can be classified into two categories, the first is to investigate pilot assignment schemes for alleviating pilot contamination $[16,17]$ and the second is devoted to the design of location-aided transmission schemes [18, 19]. Specifically, 
the authors in [16] proposed a low-complexity pilot allocation algorithm based on the line-of-sight (LOS) interference among UEs for a massive MIMO network with high-mobility UEs. In [17], a pilot allocation algorithm based on user's location was proposed, in which the pilot allocation problem can be modeled as integer quadratic constraint optimization. For the issue of transmission problem, the authors in [18] proposed a location-assisted two-layer precoding scheme based on the slow timevarying parameters for air-to-ground downlink transmission of 3-D massive MIMO. A location-aided angle-domain channel tracking and hybrid beamforming scheme was proposed in [19] for the high-speed railway (HSR) massive MIMO systems by investigating the time and spatial properties of channels.

Based on the above discussion, it is reasonable to believe that location information can play an important role in the design and optimization of cell-free massive MIMO systems. Currently, there are existing researches that have striven to design pilot assignment schemes for cell-free massive MIMO systems using location information $[20,21]$. In [20], a structured pilot assignment scheme based on location information was proposed to maximize the minimum distance between UEs assigned to the same pilot by clustering all UEs. And the authors in [21] proposed a locationbased greedy (LBG) pilot assignment scheme; the difference between the proposed LBG pilot assignment scheme and the greedy pilot assignment scheme in [1] is that the initial pilot allocation is performed according to the location before applying the greedy algorithm. To the best of our knowledge, enormous studies have been performed on location-aided communication in cellular massive MIMO systems, but not yet in cell-free massive MIMO.

\subsection{Contributions}

Motivated by these observations, we introduce location information of UEs into the design of uplink transmission scheme for user-centric cell-free massive MIMO systems in this paper, which aims to design the transmission scheme to balance the SE, UE fairness and complexity for improving the comprehensive performance of the system. To achieve the user-centric architecture, the largest-large-scale-fadingbased AP selection method is adopted. More specifically, the main contributions are listed as follows.

1) We propose a novel inter-cluster interference-based (IC-IB) pilot assignment scheme for reducing pilot contamination that takes into account the interference of UEs using the same pilot while being served by the same AP.

2) We propose a location-aided distributed uplink combining scheme. In this scheme, a new metric based on location information and the AP inter-cluster interference to indicate the degree of interference between UEs is first proposed to select UEs with more severe interference. Second, the APs that provide service to the above UEs suffering from severe interference adopt the normalized LP-MMSE combining, while the normalized MR combining is adopted for the remaining APs.

3) We derive a novel closed-form SE expression when the normalized MR combining is adopted at partial APs under this user-centric framework.

4) We propose a new metric of fairness among UEs, i.e., fairness coefficient, to compare the UE fairness of the proposed combining scheme with the existing combining schemes more fairly. 


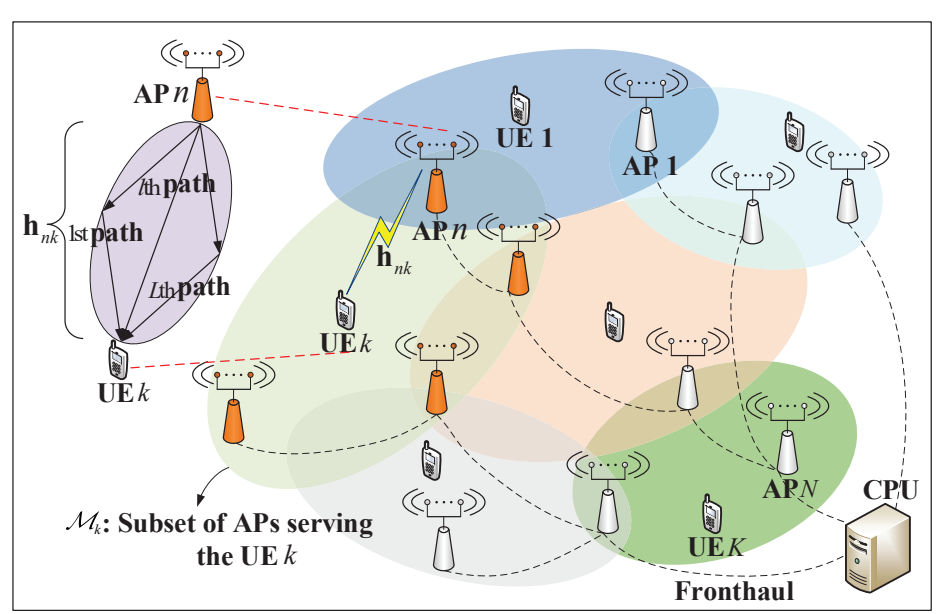

Figure 1 System Model.

5) We adopt the max-min fairness (MMF) power control algorithm to further improve the UE fairness, and compare it with the case of full power. The results demonstrate that the UE fairness for all combining schemes are improved significantly with the MMF power control. Moreover, no matter what kind of power control scheme is adopted, the proposed location-aided combining scheme has obvious advantages in comprehensive performance considering SE performance, complexity, and UE fairness.

The remainder of this paper is organized as follows. Section 2 describes the system model. Section 3 presents the proposed IC-IB pilot assignment scheme and the location-aided uplink distributed combining scheme. Additionally, the complexity analysis and the MMF power control of the proposed scheme are also discussed in this section. Section 4 provides results and discussion. Finally, the conclusions are summarized in Section 5.

Notation: Lower-case boldface symbols $\mathbf{x}$ and upper-case boldface symbols $\mathbf{X}$ denote vectors and matrices, respectively. $(\cdot)^{-1},(\cdot)^{T}$ and $(\cdot)^{H}$ denote the inverse, transpose and conjugate transpose, respectively. The expectation operation is denoted as $\mathbb{E}\{\cdot\} \cdot|\mathbf{x}|$ and $\|\mathbf{x}\|$ represent the norm and Euclidean norm, respectively, of vector $\mathbf{x}$. We also denote the cardinality and the $n$-th element of the set $\mathcal{B}$ by $|\mathcal{B}|$ and $\mathcal{B}(n)$, respectively.

\section{System Model}

We consider a user-centric cell-free massive MIMO system with $N$ APs and $K$ single-antenna UEs randomly distributed in a large area, where each UE is served by a subset of APs, as shown in Fig. 1. The different colored regions represent AP clusters serving different UEs. Notably, the AP clusters partially overlap, which is a typical feature of a user-centric cell-free architecture. Moreover, each AP is equipped with a uniform linear array (ULA) containing $M$ antenna elements and connected via an error-free fronthaul link to a CPU, which facilitates data sharing and centralized operation for resource allocation tasks between all APs. The uplink transmission is considered in this paper, and each coherence block consists of $\tau_{c}$ 
samples, where $\tau_{p}$ is used for uplink pilots and $\tau_{c}-\tau_{p}$ is used for uplink data transmission.

\subsection{Channel Model}

We assume that there are numerous scatterers in the coverage area, and the propagation of a signal from an AP to a UE is composed of $L$ paths. From the narrowband multipath transmission model, the $M \times 1$ channel vector between the $n$-th AP and the $k$-th UE is given by $[22,23]$

$$
\mathbf{h}_{n k}=\sqrt{\frac{1}{L}} \sum_{l=1}^{L} \sqrt{\beta_{n k}} \alpha_{n k}^{l} \mathbf{a}\left(\theta_{n k}^{l}\right)
$$

where $\alpha_{n k}^{l}$ is the complex gain of the $l$-th path distributed as $\alpha_{n k}^{l} \sim \mathcal{N}_{\mathbb{C}}(0,1)$ and $\mathbf{a}\left(\theta_{n k}^{l}\right)$ is the array steering vector for path $l$, which can be expressed as

$$
\mathbf{a}\left(\theta_{n k}^{l}\right)=\left[1, e^{-j \frac{2 \pi d}{\lambda} \cos \theta_{n k}^{l}}, \cdots, e^{-j \frac{2 \pi(M-1) d}{\lambda} \cos \theta_{n k}^{l}}\right]^{T}
$$

where $\theta_{n k}^{l}$ is the angle of arrival (AOA) of the $l$-th path from the $n$-th AP to the $k$-th UE, $\lambda$ is the signal wavelength, and $d$ denotes the antenna spacing, which is usually assumed to be fixed. $\beta_{n k}$ is the large-scale fading coefficient defined as [1]

$$
\beta_{n k}[\mathrm{~dB}]=\left\{\begin{array}{cc}
-81.2, & d_{n k}<10 \mathrm{~m} \\
-61.2-20 \log _{10}\left(\frac{d_{n k}}{1 \mathrm{~m}}\right), 10 \mathrm{~m} \leqslant d_{n k}<50 \mathrm{~m} \\
-35.7-35 \log _{10}\left(\frac{d_{n k}}{1 \mathrm{~m}}\right)+\delta_{n k}, & d_{n k} \geqslant 50 \mathrm{~m}
\end{array}\right.
$$

where $d_{n k}$ denotes the horizontal distance between AP $n$ and UE $k$, and $\delta_{n k} \sim$ $\mathcal{N}_{\mathbb{C}}\left(0, \sigma_{\delta_{n k}}^{2}\right)$ is the shadowing noise, where $\sigma_{\delta_{n k}}=8 \mathrm{~dB}$.

\subsection{Access Point Selection}

In traditional cell-massive MIMO systems, all UEs are assumed to be served by all APs, which is unnecessary in a large-scale network. In practice, only some APs close to the UE can provide the corresponding service; this setup is called a usercentric approach. Although this approach inevitably results in performance loss, the reduced complexity of this framework is more conducive to the network deployment.

Considering that the service quality of UEs is affected mainly by large-scale fading, we adopt the largest-large-scale-fading-based AP selection method in this paper similar to [24]. The main idea of this method is to select the APs with the largest large-scale fading coefficient to serve the corresponding UE under a given threshold, which can be mathematically expressed as

$$
\frac{\sum_{n \in \mathcal{M}_{k}} \tilde{\beta}_{n k}}{\sum_{n=1}^{N} \tilde{\beta}_{n k}} \geqslant \varepsilon
$$

where $\mathcal{M}_{k}$ denotes the subset of APs serving UE $k, \tilde{\beta}_{n k} \in\left\{\tilde{\beta}_{1 k}, \cdots, \tilde{\beta}_{N k}\right\}$ is an element of the set after sorting $\beta_{n k}, n \in\{1, \cdots, N\}$ in descending order, $0<\varepsilon<1$ is the predefined threshold for the UE to select APs. 
The main system parameters are summarized in Table 1.

Table 1 System Parameters.

\begin{tabular}{l|l}
\hline Number of APs & $N$ \\
Total number of UEs & $K$ \\
Number of antennas per AP & $M$ \\
Coherence block & $\tau_{c}$ \\
Number of uplink pilots samples & $\tau_{p}$ \\
Channel vector for the $n$-th AP and $k$-th UE & $\mathbf{h}_{n k}$ \\
Number of paths & $L$ \\
Large-scale fading coefficient & $\beta_{n k}$ \\
Small-scale fading coefficient for the $l$-th path & $\alpha_{n k}^{l}$ \\
Array steering vector for the $l$-th path & $\mathbf{a}\left(\theta_{n k}^{l}\right)$ \\
Predefined threshold for AP selection & $\varepsilon$ \\
\hline
\end{tabular}

\section{Methods}

\subsection{Inter-Cluster Interference-Based Pilot Assignment and Channel Estimation}

In the uplink pilot training phase, we assume that a set of $\tau_{p}$ mutually orthogonal uplink pilot sequences $\phi_{1}, \cdots, \phi_{\tau_{p}}$ are assigned to $K$ UEs, where $\phi_{t} \in \mathbb{C}^{\tau_{p} \times 1}$ and $\left\|\phi_{t}\right\|^{2}=\tau_{p}$, for $t \in\left\{1, \cdots, \tau_{p}\right\}$, with $\tau_{p}$ being the number of uplink training samples in each coherence interval. Due to the limited length of the coherence interval, the practical scenario is a large network with $K>\tau_{p}$ so that a pilot sequence may be assigned to multiple different UEs. Such pilot reuse among different UEs may lead to a decrease in the channel estimation accuracy, known as pilot contamination. To mitigate pilot contamination effect, we propose a novel pilot assignment scheme based on inter-cluster interference. The main idea of this pilot assignment scheme is that orthogonal pilots are assigned to the UEs with large values according to the number of common APs serving UEs, where the number of common APs can indicate the degree of interference between AP cluster. Since the more common serving APs, the interference between UEs is more serious. Subsequently, pilots are assigned to the remaining UEs based on the large-scale fading coefficient and the service relationship between the AP and UE. Specifically, the detailed steps of the proposed IC-IB pilot assignment scheme are presented as follows:

1) The CPU structures a matrix $\mathbf{S} \in \mathbb{R}^{K \times K}$ that represents the reuse of APs that provide service for different UEs based on $\left\{\mathcal{M}_{k}\right\}$, where the element of $\mathbf{S}$ is given by

$$
\mathbf{S}_{i j}=\left\{\begin{array}{cc}
\left|\mathcal{M}_{i} \cap \mathcal{M}_{j}\right|, & \text { if } i \neq j \\
\infty, & \text { else }
\end{array} .\right.
$$

The non-diagonal elements of $\mathbf{S}$ represent the number of common APs serving UEs, and $\mathbf{S}$ is a symmetric matrix; thus, we focus on the $K(K-1) / 2$ entries above the main diagonal.

2) The CPU extracts the upper triangular elements of $\mathbf{S}$ and sorts them in descending order, denoted as $\left\{\tilde{\mathbf{S}}_{i j}^{r}: i<j, r \in\{1, \cdots, K(K-1) / 2\}\right\}$. According to the sorting results, the corresponding index of UEs can be listed nonrepetitively. The first $\tau_{p}$ values are placed in index set $\mathcal{F}$, while the remaining values are placed in index set $\mathcal{S}$. Then, we assign $\tau_{p}$ orthogonal pilots to the UEs whose indices are elements in set $\mathcal{F}$. In order to facilitate the understanding, we present a simple example shown in Fig. 2, where $N=6, K=4$ and 


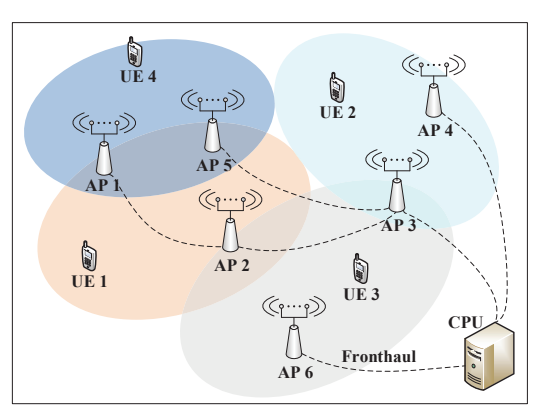

(a)

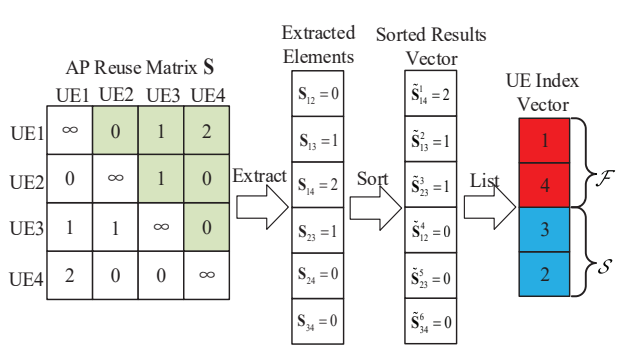

(b)

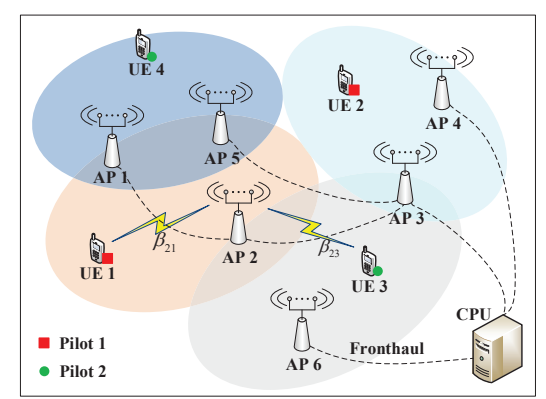

(c)

Figure 2 An example for elaborating the proposed IC-IB pilot assignment scheme. (a) The system model after AP selection; (b) Illustration of step 2 for the IC-IB pilot assignment scheme; (c) Illustration of step 3 for the IC-IB pilot assignment scheme.

$\tau_{p}=2$. More specifically, the AP reuse matrix $\mathbf{S}$ is constructed first according to the AP selection results shown in Fig. 2(a). From Fig. 2(b), the elements marked in green in matrix $\mathbf{S}$ are the elements to be extracted. The extracted elements are sorted in descending order to obtain a sorted results vector. Next, we list the index of UEs represented by the subscripts in the lower right corner one after another non-repetitively according to the sorted results $\tilde{\mathbf{S}}_{i j}^{r}$ and obtain the UE index vector. The first $\tau_{p}=2$ elements are placed into index set $\mathcal{F}$, and the UEs in $\mathcal{F}$ are assigned $\tau_{p}$ orthogonal pilots. This due to the number of common serving APs between UE 1 and UE 4 is large, which means that the interference among them is serious. Hence, the severe pilot contamination would be caused if they share the same pilot.

3) For the remaining UE $k^{\prime}, k^{\prime} \in \mathcal{S}$, when it uses the pilot $t, t \in\left\{1, \cdots, \tau_{p}\right\}$ one after another, we first calculate the sum of the average channel gains between 
the APs serving UE $k^{\prime}$ and the UEs that have already been assigned pilot $t$ and served by these APs. Then, the pilot with the least interference at the APs in the cluster serving UE $k^{\prime}$ can be found and assigned to UE $k^{\prime}$. In this example, we find the APs serving UE 3 first, i.e., AP 2, AP 3 and AP 6, and the UEs except for UE 3 served by these APs, where AP 2 and AP 3 serve UE 1 and UE 2 respectively. Then, we compute the sum of the average channel gains between the APs serving UE 3 and the UEs served by these APs that use the same pilot as UE 3. For better elaboration, we present the process of pilot assignment for UE 3 in Fig. 2(c). As seen in Fig. 2(c), pilot 1 and pilot 2 are assumed to assign UE 1 and UE 4 respectively. Note that UE 2 has not been assigned a pilot at this time. If UE 3 is assigned the pilot 1 , i.e., $t=1$, based on the step 3 mentioned above, we compute the sum of the average channel gain between AP 2 and UE 1 and that of AP 2 and UE 3, i.e., $\beta_{21}+\beta_{23}$. Similarly, when $t=2$, the same calculation method is adopted and we can obtain the result is 0 . This because the UE who has the common serving APs with UE 3 (i.e., UE 1 and UE 2) is not using pilot 2. Then, the obtained calculation results are compared and the pilot corresponding to the minimum value is assigned to UE 3 , which in this example is pilot 2. By following the same method, the pilot 1 is assigned to UE 2.

The IC-IB pilot assignment is summarized in Algorithm 1.

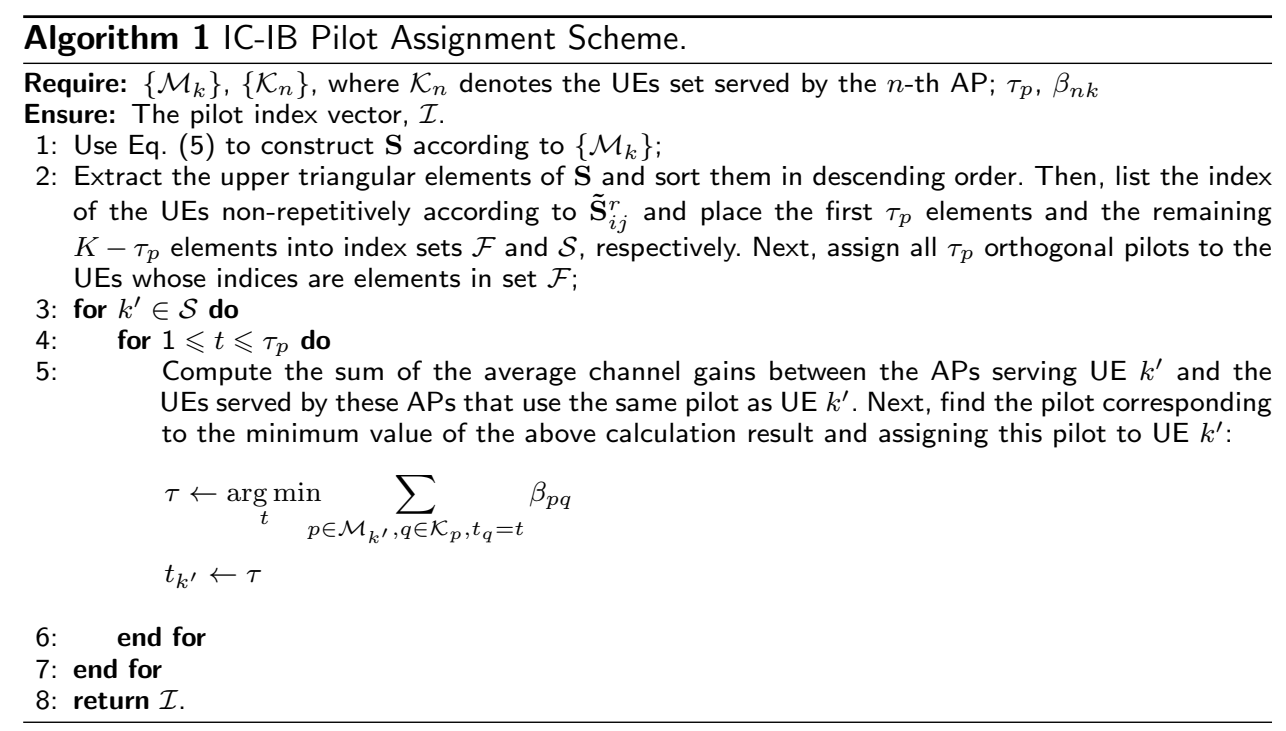

To further mitigate the interference among UEs caused by pilot reuse, we adopt the minimum mean-square-error (MMSE) channel estimation method. According to [25], the MMSE estimate $\hat{\mathbf{h}}_{n k}$ can be given by

$$
\hat{\mathbf{h}}_{n k}=\sqrt{P_{k}} \mathbf{R}_{n k} \boldsymbol{\Phi}_{n k} \mathbf{y}_{n k}^{p}
$$

where $P_{k}$ denotes the transmit power of UE $k, \mathbf{R}_{n k}=\mathbb{E}\left\{\mathbf{h}_{n k} \mathbf{h}_{n k}^{H}\right\}$ denotes the correlation matrix of channel $\mathbf{h}_{n k}, \mathbf{y}_{n k}^{p}$ is the inner product of the received signal of AP $n$ with the pilot $\phi_{k}$, and $\boldsymbol{\Phi}_{n k}=\left(\sum_{i \in \mathcal{P}_{k}} P_{i} \tau_{p} \mathbf{R}_{n i}+\mathbf{I}_{M} \sigma^{2}\right)^{-1}$, in which $\mathcal{P}_{k}$ denotes the set of UEs that use the same pilot as UE $k$. Moreover, the estimation error $\tilde{\mathbf{h}}_{n k}=$ $\mathbf{h}_{n k}-\hat{\mathbf{h}}_{n k}$ has the correlation matrix $\mathbf{C}_{n k}=\mathbb{E}\left\{\tilde{\mathbf{h}}_{n k} \tilde{\mathbf{h}}_{n k}^{H}\right\}=\mathbf{R}_{n k}-P_{k} \tau_{p} \mathbf{R}_{n k} \boldsymbol{\Phi}_{n k} \mathbf{R}_{n k}$. 


\subsection{Location-Aided Uplink Data Transmission}

In this subsection, we focus on uplink data transmission and propose a novel location-aided uplink combining scheme, which is described in detail in the following.

\subsubsection{Distributed Uplink Transmission}

During uplink data transmission, the received signal $\mathbf{y}_{n}^{u} \in \mathbb{C}^{M}$ at the $n$-th AP is given by

$$
\mathbf{y}_{n}^{u}=\sum_{i=1}^{K} \mathbf{h}_{n i} s_{i}+\mathbf{n}_{n}
$$

where $s_{i}$ is the transmitted signal from UE $i$ with power $P_{i}, \mathbf{n}_{n} \sim \mathcal{N}_{\mathbb{C}}\left(\mathbf{0}_{M}, \mathbf{I}_{M} \sigma^{2}\right)$ is the received noise at the $\operatorname{AP} n$. The distributed uplink transmission is considered in this paper. Specifically, AP $n$ first selects the combining vector $\mathbf{v}_{n k}$ and calculates the local data estimate $\hat{s}_{n k}$ by using the local channel estimates. Then, the local data estimate $\hat{s}_{n k}$ is sent to the CPU for final decoding. Under the user-centric architecture, $\hat{s}_{n k}$ can be mathematically expressed as

$$
\hat{s}_{n k}=\mathbf{v}_{n k}^{H} \mathbf{D}_{n k} \mathbf{y}_{n}^{u},
$$

where $\mathbf{D}_{n k}=\left\{\begin{array}{ll}\mathbf{I}_{M}, & k \in \mathcal{K}_{n} \\ \mathbf{0}_{M}, & k \notin \mathcal{K}_{n}\end{array}\right.$ is the matrix denoting which UEs are served by which APs.

Next, the local data estimates of the APs serving UE $k$ are collected at the CPU, after which the CPU calculates the final estimate by performing the following linear combination:

$$
\begin{aligned}
\hat{s}_{k} & =\sum_{n \in \mathcal{M}_{k}} \hat{s}_{n k}=\sum_{n \in \mathcal{M}_{k}} \mathbf{v}_{n k}^{H} \mathbf{D}_{n k}\left(\sum_{i=1}^{K} \mathbf{h}_{n i} s_{i}+\mathbf{n}_{n}\right) \\
& =\sum_{n \in \mathcal{M}_{k}} \mathbf{v}_{n k}^{H} \mathbf{D}_{n k} \mathbf{h}_{n k} s_{k}+\sum_{n \in \mathcal{M}_{k}} \sum_{i=1, i \neq k}^{K} \mathbf{v}_{n k}^{H} \mathbf{D}_{n k} \mathbf{h}_{n i} s_{i} \\
& +\sum_{n \in \mathcal{M}_{k}} \mathbf{v}_{n k}^{H} \mathbf{D}_{n k} \mathbf{n}_{n} .
\end{aligned}
$$

By using the same use-and-then-forget bound as described in [7], the achievable uplink SE of UE $k$ can be calculated as

$$
R_{k}^{u}=\frac{\tau_{c}-\tau_{p}}{\tau_{c}} \log _{2}\left(1+\operatorname{SINR}_{k}\right)
$$

where $\mathrm{SINR}_{k}$ is given by

$$
=\frac{P_{k}\left|\sum_{n \in \mathcal{M}_{k}} \mathbb{E}\left\{\mathbf{v}_{n k}^{H} \mathbf{D}_{n k} \mathbf{h}_{n k}\right\}\right|^{2}}{\sum_{i=1}^{K} P_{i} \mathbb{E}\left\{\left|\sum_{n \in \mathcal{M}_{k}} \mathbf{v}_{n k}^{H} \mathbf{D}_{n k} \mathbf{h}_{n i}\right|^{2}\right\}-P_{k}\left|\sum_{n \in \mathcal{M}_{k}} \mathbb{E}\left\{\mathbf{v}_{n k}^{H} \mathbf{D}_{n k} \mathbf{h}_{n k}\right\}\right|^{2}+\sigma^{2} \sum_{n \in \mathcal{M}_{k}} \mathbb{E}\left\{\left\|\mathbf{v}_{n k}^{H} \mathbf{D}_{n k}\right\|^{2}\right\}}
$$




\subsubsection{Existing Distributed Combining Scheme}

The core of the distributed uplink detection is the selection of the combining vector. Note that only the channel estimates for partial UEs, i.e., $k \in \mathcal{K}_{n}$, is available at the AP $n$. Therefore, only the combining vectors computed by local channel estimates and statistics can be utilized in this paper, which are usually scalable, that is, their computational complexity will not increase with the increase of the number of UEs in the whole network. Consequently, this type of combining schemes is also widely used in user-centric cell-free massive MIMO systems, which is closer to the design and deployment of the actual network. Several existing distributed combining schemes of this type are introduced in the following.

The simplest combining scheme is MR combining, which can maximize the power of the desired signal but neglects the existence of interference between UEs, given by

$$
\mathbf{v}_{n k}^{\mathrm{MR}}=\mathbf{D}_{n k} \hat{\mathbf{h}}_{n k}
$$

Eq. (12) reveals that the MR combining scheme has a low computational complexity since the combining vector is the corresponding local channel estimate and no additional computation is needed. Furthermore, uniformly good service can also be provided by the MR combining. Nevertheless, the SE performance of the system cannot be effectively guaranteed.

The second combining scheme is the normalized MR (NMR) combining. The combining vector consists of the channel estimate normalized by its magnitude, which is given by

$$
\mathbf{v}_{n k}^{\mathrm{NMR}}=\hat{\mathbf{h}}_{n k} /\left\|\hat{\mathbf{h}}_{n k}\right\|
$$

Note that the NMR combining is obtained from the normalized conjugate beamforming (NCB) scheme [26] according to the uplink-downlink duality [25] and it retains the advantages of low complexity of MR scheme, and hardens the effective channel gains at the UE to improve SE performance.

RZF is an effective combining due to it can balance between suppressing interference and maintaining strong desired signal powers. The RZF combining for UE $k$ at $\operatorname{AP} n$ is

$$
\mathbf{v}_{n k}^{\mathrm{RZF}}=P_{k}\left(\sum_{i \in \mathcal{K}_{n}} P_{i} \hat{\mathbf{h}}_{n i} \hat{\mathbf{h}}_{n i}^{H}+\mathbf{I}_{M} \sigma^{2}\right)^{-1} \mathbf{D}_{n k} \hat{\mathbf{h}}_{n k}
$$

Since there are operations such as the inverse of the matrix in the combining vector, the complexity of this scheme is higher than that of the MR and NMR combining schemes.

LP-MMSE combining is another common distributed uplink combining scheme, which is given by $[7,11]$

$$
\mathbf{v}_{n k}^{\mathrm{LP}-\mathrm{MMSE}}=P_{k}\left(\sum_{i \in \mathcal{K}_{n}} P_{i}\left(\hat{\mathbf{h}}_{n i} \hat{\mathbf{h}}_{n i}^{H}+\mathbf{C}_{n i}\right)+\mathbf{I}_{M} \sigma^{2}\right)^{-1} \mathbf{D}_{n k} \hat{\mathbf{h}}_{n k} .
$$


The LP-MMSE combining scheme can effectively utilize the local channel estimates and statistics and maximize the SINR by finding the optimal balance between amplifying the desired signal and simultaneously suppressing interference. It can be seen that the the RZF is a simplified version of the LP-MMSE since the item for covariance matrix of channel estimation error is omitted. Compared with the MR, NMR and RZF combining, the LP-MMSE combining achieves better performance but at the cost of an increased complexity. At the same time, it cannot provide the better UE fairness [11].

\subsubsection{Proposed Combining Scheme}

To achieve an effective balance between the SE performance, UE fairness and complexity, we propose a location-aided combining, and its operation steps are as follows:

1) The CPU first calculates the distances $\left\{d_{i j}: i, j \in\{1, \cdots, K\}, i<j\right\}$ between the UEs according to the obtained location information.

2) Next, we define a novel metric that indicates the degree of interference between UEs based on the distance and the number of common serving APs:

$$
\Lambda_{i j}=\omega / d_{i j}+\mathbf{S}_{i j}
$$

where $\omega$ denotes the predefined coefficient that balances the relationship between the two metrics. The novel metric described above can be computed and sorted in descending order to obtain the sorted results $\left\{\tilde{\Lambda}_{i j}^{r}: i<j, r \in\{1, \cdots, K(K-1) / 2\}\right\}$. Then, we define the set $\mathcal{G}=\emptyset$ in preparation for the following steps.

3) For $\tilde{\Lambda}_{i j}^{r}, r=1, \cdots, K(K-1) / 2$, we can find the UE corresponding to $\tilde{\Lambda}_{i j}^{r}$ and the APs that provide service to these UEs. Then, the above AP index can be inserted into set $\mathcal{G}$ one after another until $|\mathcal{G}| / N \geqslant \gamma$ holds, where $0 \leqslant \gamma \leqslant 1$ denotes the predefined threshold.

4) According to the obtained set $\mathcal{G}$ in the previous step, the APs in set $\mathcal{G}$ adopt the normalized LP-MMSE combining scheme, while the remaining APs adopt the normalized MR combining scheme, which can be expressed as

$$
\mathbf{v}_{n k}^{\mathrm{LA}}=\left\{\begin{array}{cr}
\mathbf{v}_{n k}^{\mathrm{LP}-\mathrm{MMSE}} /\left\|\mathbf{v}_{n k}^{\mathrm{LP}}-\mathrm{MMSE}\right\|, & n \in \mathcal{M}_{k}, n \in \mathcal{G} \\
\mathbf{v}_{n k}^{\mathrm{MR}} /\left\|\mathbf{v}_{n k}^{\mathrm{MR}}\right\|, & n \in \mathcal{M}_{k}, n \notin \mathcal{G}
\end{array} .\right.
$$

The location-aided combining is given in Algorithm 2.

For the proposed location-aided combining scheme, the APs that provides service to UEs are divided into two parts, one of which is detected by adopting the normalized LP-MMSE combining, while the other by the normalized MR combining. Note that the closed-form of the expectations in (11) cannot be computed when the AP using the normalized LP-MMSE combining, but the expectations can be obtained by using Monte Carlo simulations easily. However, the expectations in (11) can be computed in closed-form when the AP using the normalized MR combining, which is derived in the following. 


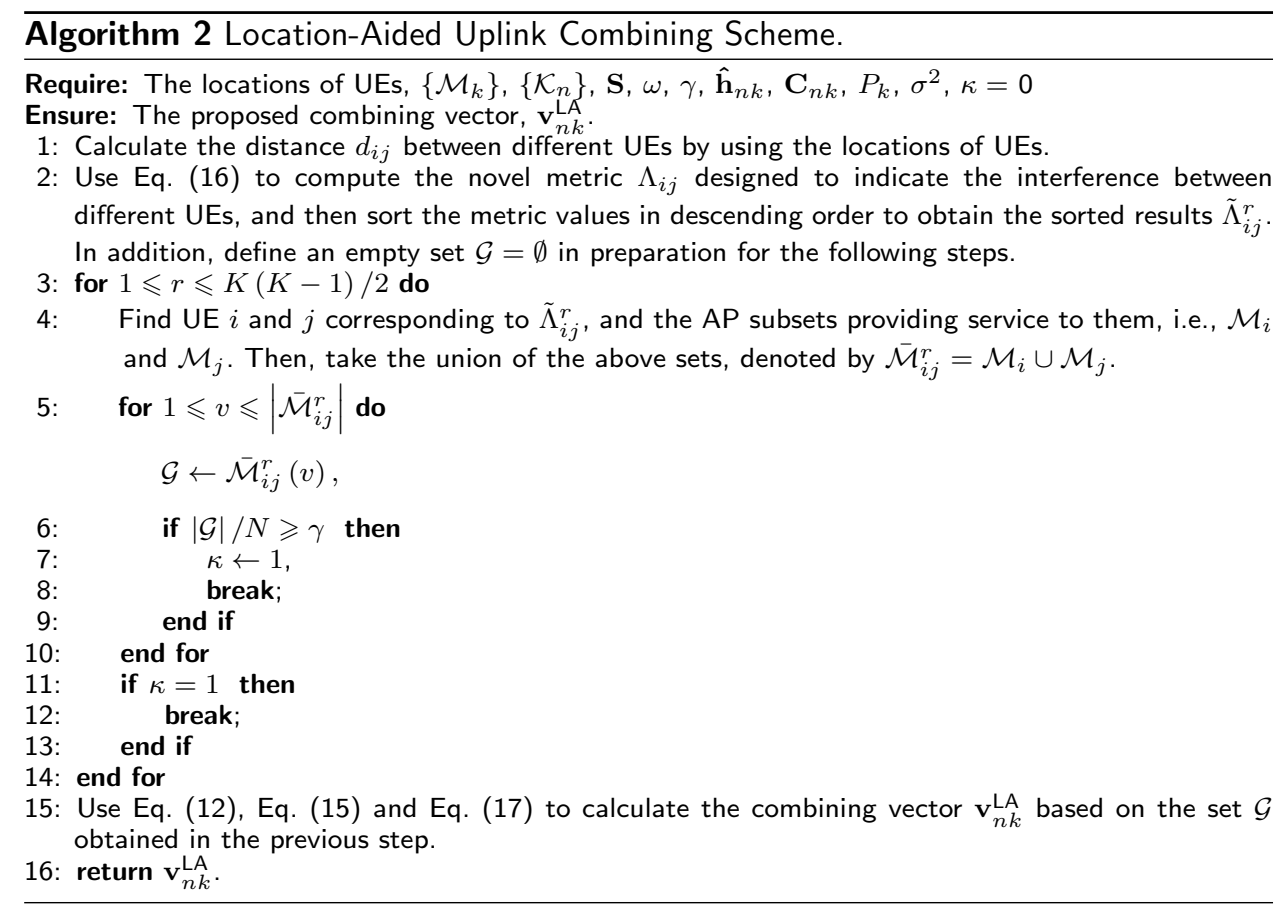

Corollary : If the APs adopt the normalized $M R$ combining, i.e., $\mathbf{v}_{n k}=$ $\hat{\mathbf{h}}_{n k} /\left\|\hat{\mathbf{h}}_{n k}\right\|$ with $n \in \mathcal{M}_{k}, n \notin \mathcal{G}$, then the expectations in (11) can be given by

$$
\mathbb{E}\left\{\mathbf{v}_{n k}^{H} \mathbf{D}_{n k} \mathbf{h}_{n k}\right\}=P_{k} \tau_{p} \operatorname{tr}\left(\mathbf{D}_{n k} \mathbf{R}_{n k} \boldsymbol{\Phi}_{n k} \mathbf{R}_{n k}\right) / \Omega_{n k},
$$

where

$$
\Omega_{n k}=\mathbb{E}\left\{\left\|\hat{\mathbf{h}}_{n k}\right\|\right\}=\frac{\chi_{n k}}{\sqrt{\eta_{n k}}} \frac{\Gamma\left(\frac{\eta_{n k}^{2}}{\chi_{n k}^{2}}+\frac{1}{2}\right)}{\Gamma\left(\frac{\eta_{n k}^{2}}{\chi_{n k}^{2}}\right)},
$$

with $\chi_{n k}$ and $\eta_{n k}$ are respectively given by

$$
\begin{aligned}
& \chi_{n k}=P_{k} \tau_{p} \sqrt{\operatorname{tr}\left(\left(\mathbf{R}_{n k} \boldsymbol{\Phi}_{n k} \mathbf{R}_{n k}\right)^{2}\right)}, \\
& \eta_{n k}=P_{k} \tau_{p} \operatorname{tr}\left(\mathbf{R}_{n k} \boldsymbol{\Phi}_{n k} \mathbf{R}_{n k}\right) . \\
& \mathbb{E}\left\{\left\|\mathbf{v}_{n k}^{H} \mathbf{D}_{n k}\right\|^{2}\right\}=1, \\
& \mathbb{E}\left\{\left|\mathbf{v}_{n k}^{H} \mathbf{D}_{n k} \mathbf{h}_{n i}\right|^{2}\right\}=\frac{\operatorname{tr}\left(\mathbf{D}_{n k} \mathbf{R}_{n i} \mathbf{R}_{n k} \boldsymbol{\Phi}_{n k} \mathbf{R}_{n k}\right)}{\operatorname{tr}\left(\mathbf{R}_{n k} \boldsymbol{\Phi}_{n k} \mathbf{R}_{n k}\right)} \\
& +\left\{\begin{array}{cc}
\frac{P_{i} \tau_{p}\left|\operatorname{tr}\left(\mathbf{D}_{n k} \mathbf{R}_{n i} \mathbf{\Phi}_{n k} \mathbf{R}_{n k}\right)\right|^{2}}{\operatorname{tr}\left(\mathbf{R}_{n k} \mathbf{\Phi}_{n k} \mathbf{R}_{n k}\right)}, & \text { if } i \in \mathcal{P}_{k} \\
0, & \text { otherwise }
\end{array}\right.
\end{aligned}
$$

Proof The proof is given in Appendix A. 


\subsection{Complexity Analysis}

In this subsection, we present complexity analysis for different distributed combining schemes. The complexity of a combining scheme originates mainly from the calculation of the combining vector. This is because the acquisition of the estimated channel is the same for each combining scheme, and the computational complexity in this process is not taken into consideration. Next, we focus on the computational complexity of the combining vector for the proposed combining scheme.

More specifically, recalling that all APs are divided into two parts for the proposed combining scheme, hence the computational complexity of the proposed combining scheme should be analyzed with regard to these two aspects respectively.

Proposition 1: The computational complexity of the location-aided uplink combining scheme is given by

$$
C^{\mathrm{LA}}=\sum_{n \in \mathcal{G}} \frac{M^{2}+M}{2}\left|\mathcal{K}_{n}\right|+\frac{M^{3}-M}{3}+M^{2}\left|\mathcal{K}_{n}\right|+M\left|\mathcal{K}_{n}\right|+\sum_{n^{\prime} \in \mathcal{A} \backslash \mathcal{G}}\left(M\left|\mathcal{K}_{n^{\prime}}\right|\right) \cdot \mathbb{I}\left(\mathcal{K}_{n^{\prime}} \neq \emptyset\right)
$$

where $\mathcal{A}$ denotes the set of all $A P s, \mathcal{A} \backslash \mathcal{G}$ represents the set of APs except the elements in set $\mathcal{G}$, and $\mathbb{I}(E)$ is one if the logic statement $E$ is true.

Proof The combining vector is computed by using elementary matrix operations, such as matrix-matrix multiplication and matrix inversion. Therefore, we can calculate the computational complexity with the help of the framework shown in [25, Appendices. B.1.1], where only complex multiplications and divisions are considered, while additions and subtractions can be neglected. Specifically, for the APs adopting the normalized LP-MMSE combining scheme, we can first compute the number of multiplications $\frac{M^{2}+M}{2}\left|\mathcal{K}_{n}\right|$ for the term $\hat{\mathbf{h}}_{n i} \hat{\mathbf{h}}_{n i}^{H}$ with $i \in \mathcal{K}_{n}$ resorting to [25, Lemma B.1.]. Then, based on [25, Lemma B.2.], $\left(\sum_{i \in \mathcal{K}_{n}} P_{i}\left(\hat{\mathbf{h}}_{n i} \hat{\mathbf{h}}_{n i}^{H}+\mathbf{C}_{n i}\right)+\mathbf{I}_{M} \sigma^{2}\right)$ can be performed $\mathbf{L D L}^{H}$ decomposition to achieve an efficient hardware implementation, which can be calculated using $\frac{M^{3}-M}{3}$ complex multiplications. Next, the inverse of the above matrix is multiplied by $\mathbf{D}_{n k} \hat{\mathbf{h}}_{n k}$, which requires $M^{2}\left|\mathcal{K}_{n}\right|$ multiplications. Ultimately, the number of complex multiplications $M\left|\mathcal{K}_{n}\right|$ for calculating the vector norm can be obtained. On the other hand, for the remaining APs, when the AP serves at least one UE, the normalized MR combining is adopted. Since only the channel estimates are used for signal combining in this case, no additional calculations are required except for the calculation of the vector norm. Therefore, the complexity is also computed as $M\left|\mathcal{K}_{n^{\prime}}\right|$. Based on the above analysis, the computational complexity of the proposed scheme in Proposition $\mathbf{1}$ can be easily derived.

Remark 1: Note that Eq. (24) does not consider the calculation of distances between UEs. The main reasons for this are as follows. First, the calculation amount of distances between UEs are smaller than those of the combining vector. Second, the locations of UEs can be treated as unchanged within several or even tens of channel coherence blocks since the channel coherence time is short, whereas the position of a UE and its surrounding environment may not physically change in a comparable period of time. Hence, unlike combining vectors, it is not necessary 
to frequently calculate the distances between UEs. Based on this, the calculation of distance between UEs is negligible for the complexity of the proposed scheme.

Proposition 2: The computational complexity of the LP-MMSE, RZF, NMR and MR combining schemes are respectively given by

$$
\begin{aligned}
& C^{\mathrm{LP}-\mathrm{MMSE}}=\sum_{n=1}^{N}\left(\frac{M^{2}+M}{2}\left|\mathcal{K}_{n}\right|+\frac{M^{3}-M}{3}+M^{2}\left|\mathcal{K}_{n}\right|\right) \times \mathbb{I}\left(\mathcal{K}_{n} \neq \emptyset\right), \\
& C^{\mathrm{RZF}}=\sum_{n=1}^{N}\left(\frac{M^{2}+M}{2}\left|\mathcal{K}_{n}\right|+\frac{M^{3}-M}{3}+M^{2}\left|\mathcal{K}_{n}\right|\right) \times \mathbb{I}\left(\mathcal{K}_{n} \neq \emptyset\right), \\
& C^{\mathrm{NMR}}=\sum_{n=1}^{N}\left(M\left|\mathcal{K}_{n}\right|\right) \cdot \mathbb{I}\left(\mathcal{K}_{n} \neq \emptyset\right), \\
& C^{\mathrm{MR}}=0 .
\end{aligned}
$$

Proof The proof of the complexity for the LP-MMSE combining scheme follows the similar approach as in Proposition 1. It is worth noting that there is no $M\left|\mathcal{K}_{n}\right|$ term in Eq. (25) because there is no need to calculate the vector norm. The complexity of RZF combining is equal to the LP-MMSE combining since the change of omitting the item of covariance matrix has a negligible impact on the complexity. The complexity of NMR combining is shown in Eq. (27) because the norm of the vector needs to be computed for normalization. Moreover, the MR combining requires no additional computation; hence, the complexity is 0 .

\subsection{Max-Min Fairness Power Control}

Pragmatic power control is needed in practical implementations of cell-free massive MIMO systems. Motivated by [1] and [5], we introduce the MMF power control in this subsection, which can provide complete fairness by optimizing the SE achieved by the weakest UE in the network. The MMF power control problem can be formulated as:

$$
\begin{aligned}
& \max _{P_{k}} \min _{k \in\{1, \cdots, K\}} R_{k}^{u} \\
& \text { s.t. } 0 \leqslant P_{k} \leqslant P_{\max }, k=1, \cdots, K,
\end{aligned}
$$

where $P_{\max }$ denotes the maximum transmit power of UE. Without loss of generality, the problem given in (29) can be rewritten by introducing an auxiliary variable $w$ :

$$
\begin{aligned}
& \max _{w, P_{k}} w \\
& \text { s.t. } \mathrm{SINR}_{k} \geqslant w, k=1, \cdots, K \\
& \quad 0 \leqslant P_{k} \leqslant P_{\max }, k=1, \cdots, K .
\end{aligned}
$$


Then, to improve the convergence rate, we equivalently replace the problem in (30) with a problem having the same constraints but where the total power is minimized, which is similar to [5], given by

$$
\begin{aligned}
& \min _{P_{k}} \sum_{k=1}^{K} P_{k} \\
& \text { s.t. } \mathrm{SINR}_{k} \geqslant w, k=1, \cdots, K \\
& \quad 0 \leqslant P_{k} \leqslant P_{\max }, k=1, \cdots, K .
\end{aligned}
$$

Finally, the above problem can be efficiently solved by bisection search method, which is given in Algorithm 3.

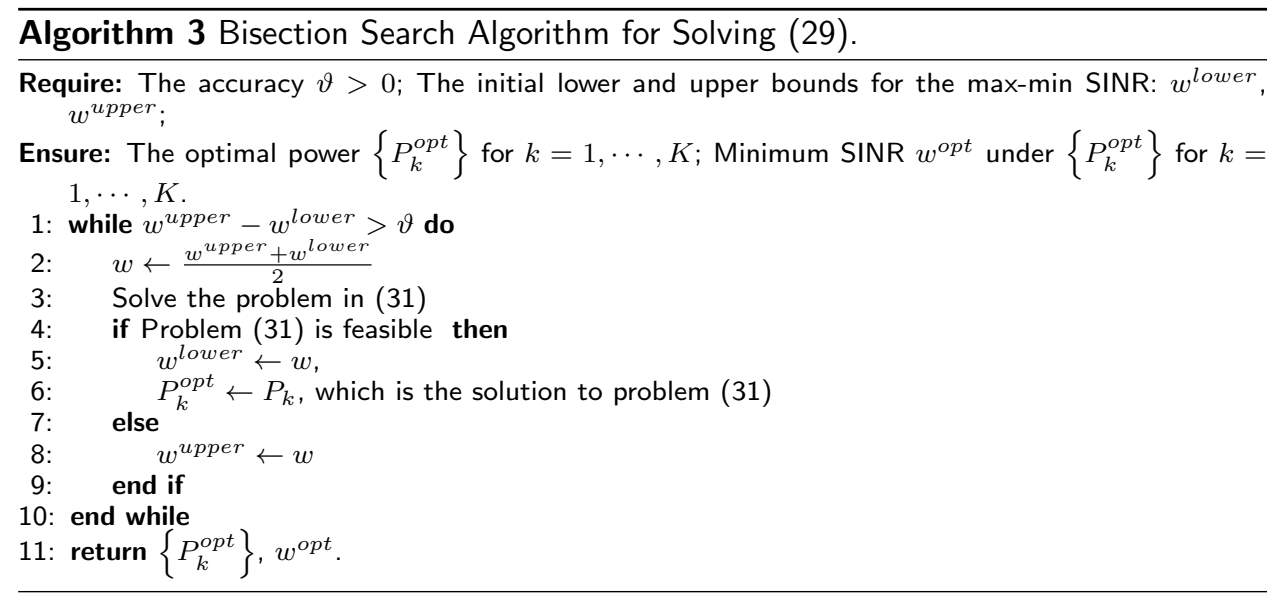

Table 2 Parameters Setup.

\begin{tabular}{l|l}
\hline Parameters & Value \\
\hline Number of UEs, $K$ & 20 \\
Number of scattering paths, $L$ & 10 \\
Noise power, $\sigma^{2}$ & $-96 \mathrm{dBm}$ \\
Single-side angle spread & $4^{\circ}$ \\
Maximum uplink transmit power, $P \max$ & $100 \mathrm{~mW}$ \\
Number of random realizations of AP/UE locations & 100 \\
Number of random channel realizations & 1000 \\
\hline
\end{tabular}

\section{Results and Discussion}

In this section, we evaluate the performance of the proposed IC-IB pilot assignment scheme in comparison with that of the existing pilot assignment schemes, and we present simulation results to investigate the performance of the location-aided uplink combining scheme in terms of the SE performance, UE fairness and computational complexity respectively. We consider a simulation scenario where all APs and UEs are randomly distributed in $300 \times 300 \mathrm{~m}^{2}$. The ULA antenna spacing is $d=\frac{\lambda}{2}$. Each coherence block contains $\tau_{c}=100$ samples, where $\tau_{p}=10$ is utilized for uplink pilot training. Unless specified, full power is used for transmission. Furthermore, the AP selection threshold and the predefined coefficient are set to $\varepsilon=$ $90 \%$ and $\omega=1000$ respectively. The other specific parameters are summarized in Table 2. 
Then, we use the mean-squared error (MSE) to evaluate the performance of channel estimation. Considering the user-centric framework adopted in this paper, the expression of MSE can be given by [27]

$$
\mathrm{MSE} \triangleq \frac{1}{K} \sum_{k=1}^{K} \frac{\sum_{n \in \mathcal{M}_{k}}\left\|\mathbf{h}_{n k}-\hat{\mathbf{h}}_{n k}\right\|^{2}}{\sum_{n \in \mathcal{M}_{k}}\left\|\mathbf{h}_{n k}\right\|^{2}} .
$$

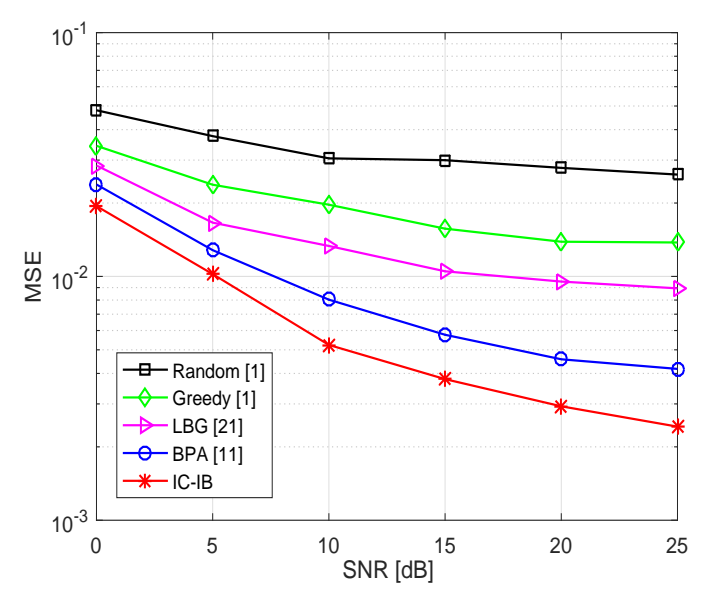

Figure $3 \mathrm{MSE}$ of channel estimation with various received signal-to-noise ratio (SNR) for different pilot assignment schemes with $M=8, N=30$.

Fig. 3 compares the MSE of the channel estimation performance versus SNR with different pilot assignment schemes. Clearly, the proposed IC-IB pilot assignment scheme is superior to the existing pilot assignment schemes such as random, greedy, LBG and basic pilot assignment (BPA) schemes. There are two reasons for this superiority. First, $\tau_{p}$ mutually orthogonal pilots are assigned to $\tau_{p}$ UEs with severe potential interference, rather than randomly. Second, for the UEs remaining after these orthogonal pilots are assigned, we consider the interference between the corresponding UE and the UEs served by the common APs that use the same pilot, and the interference relationship between UEs is used to assign pilots to the maximum extent under the user-centric framework. Furthermore, the MSE performance is improved with the increase of SNR for all schemes and we can improve the channel estimation accuracy by increasing SNR appropriately.

Fig. 4 compares per-UE uplink SE, average SE and UE fairness when different combining schemes are applied under different number of AP antennas $M$, where the UE fairness is measured by the fairness coefficient, which is inspired by the literature [28] and redefined as the ratio of the difference between the maximum and minimum $\mathrm{SE}$ and the average $\mathrm{SE}$, i.e., $\frac{\mathrm{R}_{\max }^{u}-\mathrm{R}_{\min }^{u}}{\frac{1}{K} \sum_{k=1}^{K} \mathrm{R}_{k}^{u}}$, for achieving fair comparison. More specifically, Figs. 4(a) and 4(b) reveal that under the same condition, our proposed location-aided combining outperforms the other four schemes in terms of the $90 \%$-likely per-UE SE, which means that the proposed combining scheme improves the minimum SE of UEs. Furthermore, the proportion of UEs with a higher SE of the proposed combining is lower than that of the LP-MMSE and RZF 


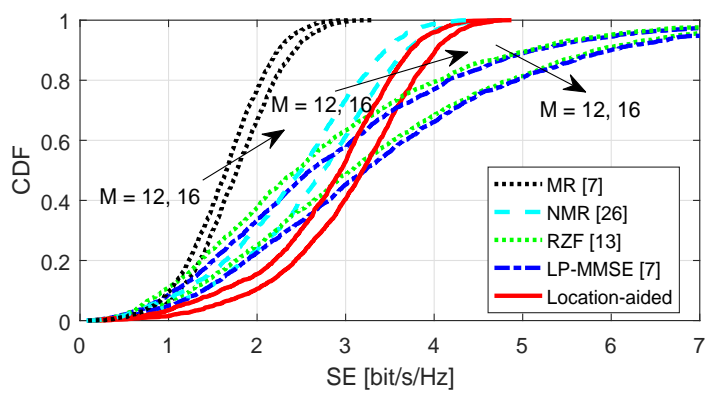

(a) $\mathrm{CDF}$ of $\mathrm{SE}$.

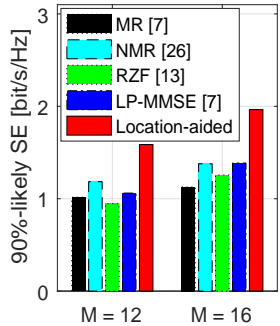

(b) $90 \%$-likely SE.

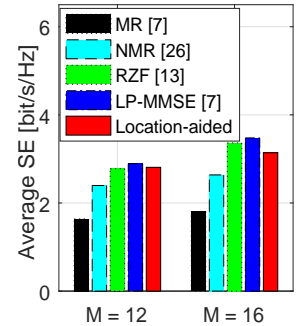

(c) Average SE.

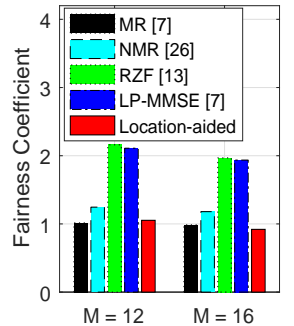

(d) UE fairness.

Figure 4 The comparison of per-UE uplink SE, average SE and UE fairness with different number of antennas for different combining schemes, where $N=30, \gamma=0.5$ and $M=12,16$.

combining schemes. This is the reason because only some of the APs in the proposed combining scheme adopt normalized LP-MMSE combining which can effectively suppress the interference between UEs. As shown in Fig. 4(c) and Fig. 4(d), in terms of average $\mathrm{SE}$, with the increase of $M$, the proposed combining is superior to the MR and NMR schemes, and exhibits only a slight performance loss compared with the LP-MMSE and RZF combining schemes. While on the aspect of UE fairness, the proposed combining outperforms the LP-MMSE and RZF combining, and achieves fairness performance similar to the MR combining scheme. Therefore, our proposed combining can provide a good tradeoff between the UE fairness and average SE. Furthermore, as shown in Fig. 4, the SE performance improves with increasing $M$ since more antennas can offer more degrees of freedom.

Fig. 5 illustrates per-UE uplink SE, average SE and UE fairness for different combining schemes with different thresholds $\gamma$. As shown in Figs. 5(a) and 5(b), the 90\%-likely per-UE SE of the proposed combining scheme outperforms those of the other four schemes even if the value of $\gamma$ is small; for example, when $\gamma=$ 0.2 , the proposed combining scheme can improve the 90\%-likely per-UE SE by $19.1 \%, 5.7 \%, 38.6 \%$ and $16.7 \%$ with respect to the MR, NMR, RZF and LP-MMSE combining schemes, respectively. As shown in Fig. 5(c) and Fig. 5(d), the average SE and UE fairness of the proposed scheme improve with the increase of $\gamma$. This is intuitive since a larger threshold $\gamma$ means that more APs use the normalized LP-MMSE combining. However, this improvement comes at the cost of an increase in computational complexity, and we should find a good compromise between the SE performance and complexity according to the actual network requirements. 


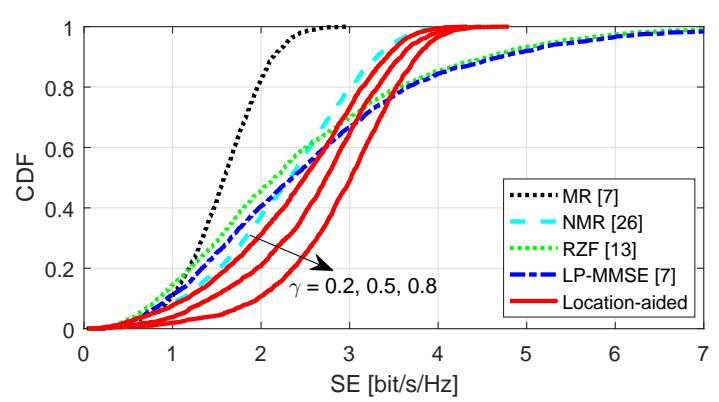

(a) CDF of SE.

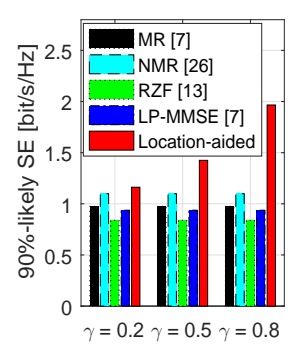

(b) $90 \%$-likely SE.

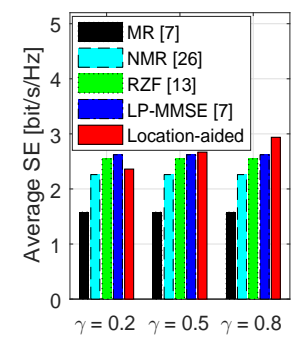

(c) Average SE.

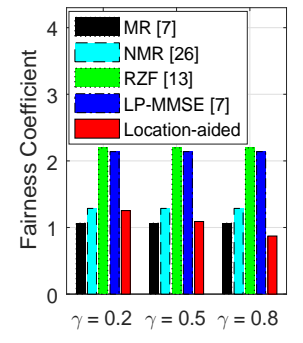

(d) UE fairness.

Figure 5 The comparison of per-UE uplink SE, average SE and UE fairness with different thresholds $\gamma$ for different combining schemes, where $M=10, N=30$ and $\gamma=0.2,0.5,0.8$.

In Fig. 6, the behavior of sum SE varying with $M$ and $N$ for different combining schemes is displayed. Clearly, the sum SE of all combining schemes monotonously increases with $M$ and $N$ since more degrees of freedom can be provided by more antennas and APs. Besides, in Fig. 6(a), the sum SE performance of the proposed combining scheme is superior to the MR and NMR schemes in the entire range of $M$ values and if $M$ is small (i.e., $M \leqslant 10$ ), it is also better than the LP-MMSE and RZF combining schemes. However, when the number of AP antennas is greater than a certain value (i.e., $M \geqslant 13$ ), both LP-MMSE and RZF schemes outperforms the proposed scheme in terms of sum SE. Nevertheless, due to the limitation of external factors such as cost, the number of AP antennas in cell-free massive MIMO systems will not be large, and the proposed location-aided combining scheme still has certain advantages when applied to cell-free massive MIMO systems. Moreover, we can also observe that the proposed combining scheme outperforms the other four schemes in terms of sum SE in Fig. 6(b), which shows that the best services can be offered by the location-aided combining scheme for the entire system under this condition.

Fig. 7 depicts the number of complex multiplications required for the computation of different combining schemes obtained from Proposition 1 and Proposition 2. Specifically, from 7(a), we observe that the complexity of our proposed combining scheme and that of the NMR, RZF and LP-MMSE combining schemes increase with $M$, whereas the complexity of the MR combining scheme remains unchanged and equals to 0. Besides, we can also see that the curve of LP-MMSE scheme coincides with RZF, and the complexity curve of NMR scheme varies slight with $M$ since there is only norm operation of vector, which are consistent with the above analysis. 


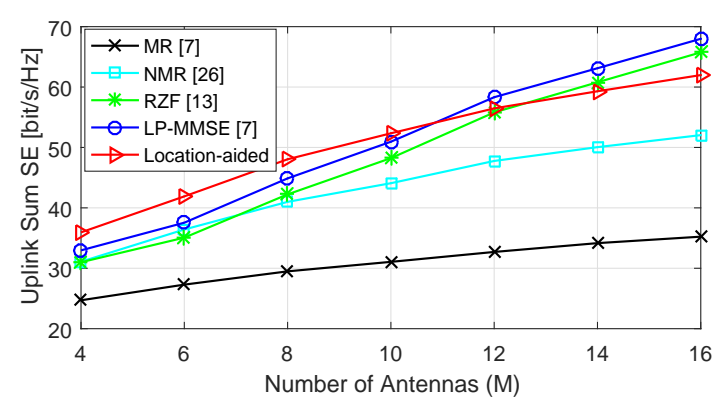

(a)

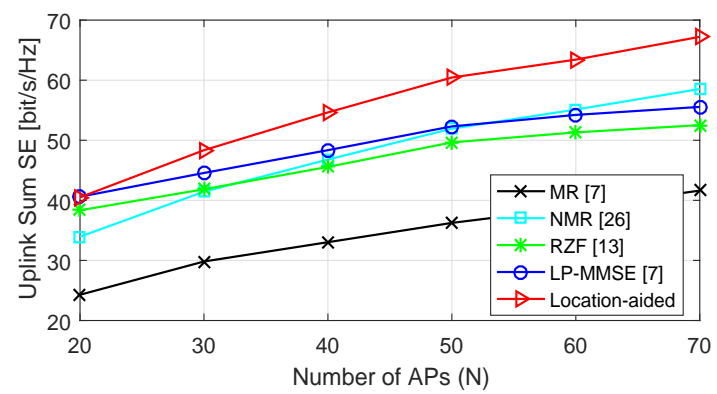

(b)

Figure 6 Uplink sum SE versus $M$ or $N$ for different combining schemes. (a) $N=30, \gamma=0.5$; (b) $M=8, \gamma=0.5$.

Moreover, our proposed scheme is superior to the LP-MMSE and RZF combining schemes in terms of the computational complexity, and the gap between the two broadens with the increase of $M$, which means that when $M$ is large, the proposed combining scheme has an advantageously lower complexity. In Fig. 7(b), the average $\mathrm{SE}$ and the number of complex multiplications of the proposed combining scheme increase with $\gamma$, whereas those of the four other schemes remain unchanged. The proposed combining scheme can select an appropriate $\gamma$ to balance the uplink SE and computational complexity according to the actual requirements of the network. Specifically, when $\gamma<0.35$ or $\gamma<0.2$, although the SE performance of the proposed combining scheme is not better than that of LP-MMSE or RZF combining schemes, its complexity is lower. In addition, when $0.35 \leqslant \gamma \leqslant 0.9$, the proposed combining scheme is superior to the LP-MMSE combining with regard to both average SE and complexity, and also outperforms RZF combining if $0.2 \leqslant \gamma \leqslant 0.9$, highlighting the advantages of our proposed location-aided combining scheme.

Fig. 8 compares per-UE uplink SE, average SE and UE fairness with MMF power control and full power for different combining schemes. In Figs. 8(a), 8(b) and $8(\mathrm{e})$, it can be seen that the $90 \%$-likely per-UE SE of the location-aided, LP-MMSE, RZF and NMR combining schemes using MMF power control is increased by $19.4 \%$, $64.4 \%, 79.2 \%$ and $43.3 \%$ respectively compared with full power. As seen in Figs. $8(\mathrm{c}), 8(\mathrm{f}), 8(\mathrm{~d})$ and $8(\mathrm{~g})$, although average SE for all combining schemes with MMF power control is inferior to that of full power, the UE fairness coefficient can be significantly decreased. That is to say, the MMF power control can greatly improve the 


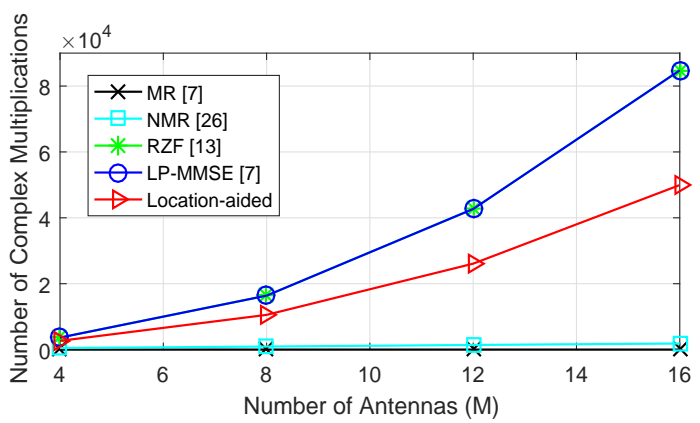

(a)

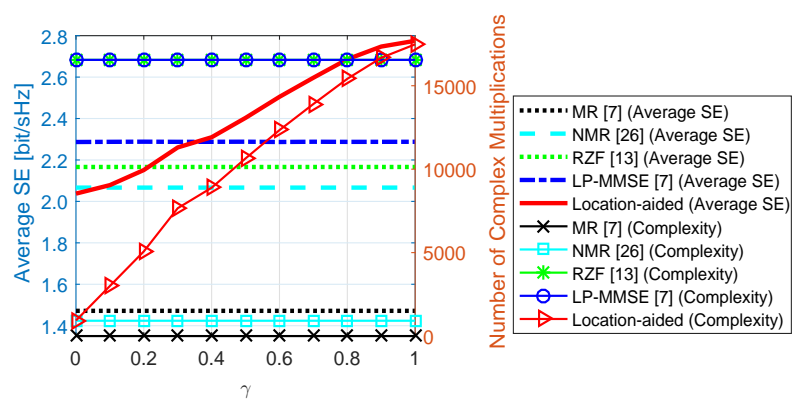

(b)

Figure 7 For different combining schemes: (a) The number of complex multiplications versus $M$, where $N=30, \gamma=0.5$; (b) Average SE and the number of complex multiplications versus the threshold $\gamma$, where $M=8, N=30$.

fairness between UEs and provide more uniform service for all UEs in the network. Furthermore, we also notice that the location-aided combining scheme achieves the similar performance in terms of the average SE and UE fairness coefficient to the LP-MMSE and RZF schemes when adopting the MMF power control, while the former is superior to the latter two in terms of complexity. When full power is adopted, the proposed scheme is superior to the other four schemes in terms of the overall performance that takes into account SE performance, complexity, and UE fairness. In other words, the proposed combining scheme has significant advantages under both two power control schemes.

\section{Conclusion}

This paper investigates the uplink transmission of cell-free massive MIMO systems based on a user-centric topology. The largest-large-scale-fading-based AP selection method is adopted, and a novel pilot assignment scheme based on the inter-cluster interference is proposed to reduce pilot contamination. Based on the location information of UEs and the service relationship of AP-UE pairs, we design a new metric to represent the degree of interference between UEs and then propose a locationaided distributed uplink combining scheme, that can select the APs served by UEs with large inter-user interference to adopt the normalized LP-MMSE combining scheme, while the normalized MR combining is adopted for the remaining APs. We also derive the closed-form SE expression when using the normalized MR combining and propose a new metric, i.e., fairness coefficient, to characterize UE fairness. 


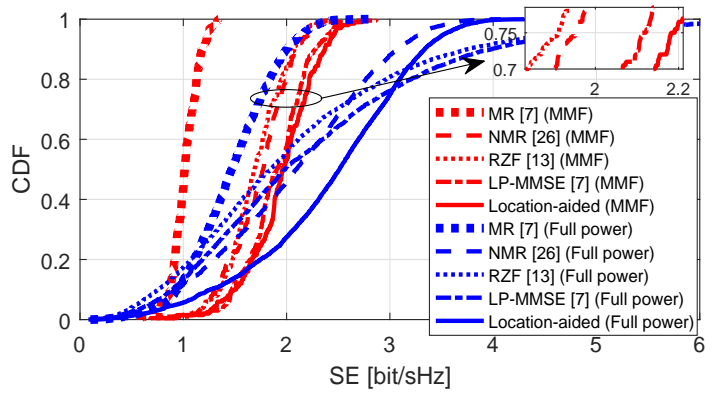

(a) CDF of SE.

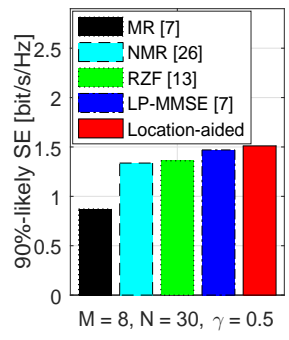

(b) $90 \%$-likely SE (MMF).

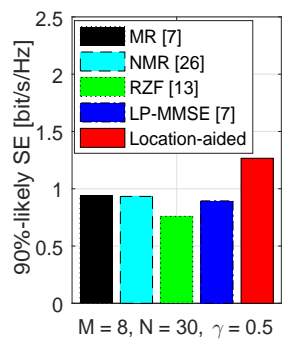

(e) $90 \%$-likely SE (full).

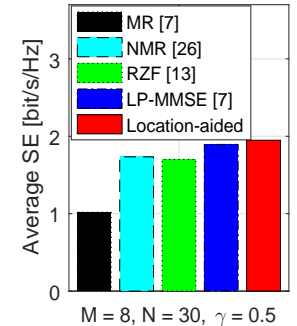

(c) Average SE (MMF).

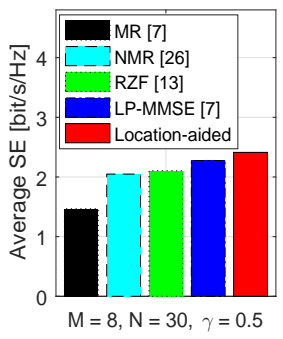

(f) Average SE (full).

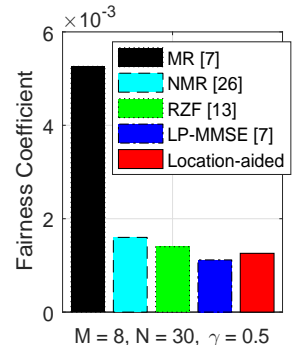

(d) UE fairness (MMF)

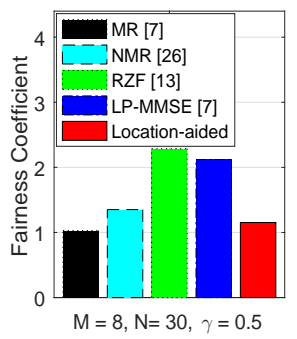

(g) UE fairness (full).

Figure 8 The comparison of per-UE uplink SE, average SE and UE fairness with different power control schemes, where $M=8, N=30$ and $\gamma=0.5$.

In order to further improve the uniformity of network services, the MMF power control algorithm is also introduced to maximize the SE achieved by the weakest UE. Simulation results show that the proposed IC-IB pilot assignment scheme achieves better channel estimation performance than the existing pilot assignment schemes. Moreover, our proposed location-aided uplink combining scheme improves the UE fairness significantly and effectively compromises between the average SE and complexity, which has obvious advantages when considering the comprehensive performance of the above three metrics in comparison to the existing combining schemes and is useful in uplink transmission design for user-centric cell-free massive MIMO systems.

\section{Appendix A}

By substituting $\mathbf{v}_{n k}=\frac{\hat{\mathbf{h}}_{n k}}{\left\|\hat{\mathbf{h}}_{n k}\right\|}$ with $n \in \mathcal{M}_{k}, n \notin \mathcal{G}$ into the expectation of the numerator in (11), we have 


$$
\begin{aligned}
& \mathbb{E}\left\{\mathbf{v}_{n k}^{H} \mathbf{D}_{n k} \mathbf{h}_{n k}\right\}=\mathbb{E}\left\{\frac{\hat{\mathbf{h}}_{n k}^{H}}{\left\|\hat{\mathbf{h}}_{n k}\right\|} \mathbf{D}_{n k} \mathbf{h}_{n k}\right\} \\
& =\mathbb{E}\left\{\frac{\hat{\mathbf{h}}_{n k}^{H}}{\left\|\hat{\mathbf{h}}_{n k}\right\|} \mathbf{D}_{n k}\left(\hat{\mathbf{h}}_{n k}+\tilde{\mathbf{h}}_{n k}\right)\right\} \\
& =\underbrace{\mathbb{E}\left\{\frac{\hat{\mathbf{h}}_{n k}^{H}}{\left\|\hat{\mathbf{h}}_{n k}\right\|} \mathbf{D}_{n k} \hat{\mathbf{h}}_{n k}\right\}}_{\Delta_{1}}+\underbrace{\mathbb{E}\left\{\frac{\hat{\mathbf{h}}_{n k}^{H}}{\left\|\hat{\mathbf{h}}_{n k}\right\|} \mathbf{D}_{n k} \tilde{\mathbf{h}}_{n k}\right\}}_{\Delta_{2}} \\
& \stackrel{(a)}{=} \mathbb{E}\left\{\frac{\hat{\mathbf{h}}_{n k}^{H}}{\left\|\hat{\mathbf{h}}_{n k}\right\|} \mathbf{D}_{n k} \hat{\mathbf{h}}_{n k}\right\} \stackrel{(b)}{\approx} \frac{\mathbb{E}\left\{\hat{\mathbf{h}}_{n k}^{H} \mathbf{D}_{n k} \hat{\mathbf{h}}_{n k}\right\}}{\mathbb{E}\left\{\left\|\hat{\mathbf{h}}_{n k}\right\|\right\}} \\
& \stackrel{(c)}{=} \frac{P_{k} \tau_{p} \operatorname{tr}\left(\mathbf{D}_{n k} \mathbf{R}_{n k} \boldsymbol{\Phi}_{n k} \mathbf{R}_{n k}\right)}{\mathbb{E}\left\{\left\|\hat{\mathbf{h}}_{n k}\right\|\right\}},
\end{aligned}
$$

where $\Delta_{2}=0$ since the channel estimate $\hat{\mathbf{h}}_{n k}$ and channel estimation error $\tilde{\mathbf{h}}_{n k}$ are independent and both have zero mean, $(a)$ follows the above operation and reserves the former term $\Delta_{1}$ as shown in equation. It is worth noting that the expectation of the matrix $\hat{\mathbf{h}}_{n k}^{H} \mathbf{D}_{n k} \hat{\mathbf{h}}_{n k} /\left\|\hat{\mathbf{h}}_{n k}\right\|$ is difficult to obtain. Hence, (b) utilizes an approximation method widely used in the calculation of the expectation of quotient, i.e., the first order Taylor approximation, to solve this issue, which is the same as that used in [29] and [30]. Next, we need to calculate the closed-form expression of $\mathbb{E}\left\{\hat{\mathbf{h}}_{n k}^{H} \mathbf{D}_{n k} \hat{\mathbf{h}}_{n k}\right\}$. Resorting to the approach as in [25, Corollary. 4.5], we have

$$
\begin{aligned}
\mathbb{E}\left\{\hat{\mathbf{h}}_{n k}^{H} \mathbf{D}_{n k} \hat{\mathbf{h}}_{n k}\right\} & \stackrel{(d)}{=} \operatorname{tr}\left(\mathbb{E}\left\{\hat{\mathbf{h}}_{n k} \mathbf{D}_{n k} \hat{\mathbf{h}}_{n k}^{H}\right\}\right) \\
& \stackrel{(e)}{=} P_{k} \tau_{p} \operatorname{tr}\left(\mathbf{D}_{n k} \mathbf{R}_{n k} \mathbf{\Phi}_{n k} \mathbf{R}_{n k}\right)
\end{aligned}
$$

where $(d)$ follows the matrix identity in [25, Lemma B.5], (e) follows the fact that $\hat{\mathbf{h}}_{n k} \sim \mathcal{N}_{\mathbb{C}}\left(\mathbf{0}_{M}, \mathbf{R}_{n k}-\mathbf{C}_{n k}\right)[25$, Corollary 3.2].

Then, we focus on the calculation of the expectation $\mathbb{E}\left\{\left\|\hat{\mathbf{h}}_{n k}\right\|\right\}$. Let us denote $\psi_{n k}=\hat{\mathbf{h}}_{n k}^{H} \hat{\mathbf{h}}_{n k}=\left\|\hat{\mathbf{h}}_{n k}\right\|^{2}$. With the help of [29, Lemma 3], the fractional $(1 / 2)$ st (non-central) moment of the Hermitian quadratic form $\psi_{n k}$ can be accurately approximated as

$$
\mathbb{E}\left\{\psi_{n k}^{1 / 2}\right\} \approx \frac{\chi_{n k}}{\sqrt{\eta_{n k}}} \frac{\Gamma\left(\frac{\eta_{n k}^{2}}{\chi_{n k}^{2}}+\frac{1}{2}\right)}{\Gamma\left(\frac{\eta_{n k}^{2}}{\chi_{n k}^{2}}\right)}
$$

where considering $\hat{\mathbf{h}}_{n k} \sim \mathcal{N}_{\mathbb{C}}\left(\mathbf{0}, P_{k} \tau_{p} \mathbf{R}_{n k} \boldsymbol{\Phi}_{n k} \mathbf{R}_{n k}\right), \eta_{n k}$ and $\chi_{n k}^{2}$ can be respectively given by

$$
\eta_{n k}=\mathbb{E}\left\{\psi_{n k}\right\}=P_{k} \tau_{p} \operatorname{tr}\left(\mathbf{R}_{n k} \boldsymbol{\Phi}_{n k} \mathbf{R}_{n k}\right)
$$




$$
\begin{aligned}
\chi_{n k}^{2} & =\mathbb{E}\left\{\left|\psi_{n k}-\mathbb{E}\left\{\psi_{n k}\right\}\right|^{2}\right\}=\mathbb{E}\left\{\left|\psi_{n k}\right|^{2}\right\}-\left|\mathbb{E}\left\{\psi_{n k}\right\}\right|^{2} \\
& =\left(P_{k} \tau_{p}\right)^{2} \operatorname{tr}\left(\left(\mathbf{R}_{n k} \boldsymbol{\Phi}_{n k} \mathbf{R}_{n k}\right)^{2}\right) .
\end{aligned}
$$

Plugging (36) and (37) into (35), and then substituting (35) into (33), we can obtain (18).

Next, the noise term in (11) can be easily computed as $\mathbb{E}\left\{\left\|\mathbf{v}_{n k}^{H} \mathbf{D}_{n k}\right\|^{2}\right\}=1$ when the normalized combining is utilized.

For the proposed combining scheme, the interference term in denominator of (11) can be rewritten as

$$
\begin{aligned}
& \mathbb{E}\left\{\left|\sum_{n \in \mathcal{M}_{k}} \mathbf{v}_{n k}^{H} \mathbf{D}_{n k} \mathbf{h}_{n i}\right|^{2}\right\} \\
& =\mathbb{E}\left\{\left|\sum_{n \in \mathcal{M}_{k}, n \notin \mathcal{G}} \mathbf{v}_{n k}^{H} \mathbf{D}_{n k} \mathbf{h}_{n i}+\sum_{n \in \mathcal{M}_{k}, n \in \mathcal{G}} \mathbf{v}_{n k}^{H} \mathbf{D}_{n k} \mathbf{h}_{n i}\right|^{2}\right\} \\
& \stackrel{(f)}{=} \underbrace{\mathbb{E}\left\{\left|\sum_{n \in \mathcal{M}_{k}, n \notin \mathcal{G}} \mathbf{v}_{n k}^{H} \mathbf{D}_{n k} \mathbf{h}_{n i}\right|^{2}\right\}}_{\Xi_{1}}+\underbrace{\mathbb{E}\left\{\left|\sum_{n \in \mathcal{M}_{k}, n \in \mathcal{G}} \mathbf{v}_{n k}^{H} \mathbf{D}_{n k} \mathbf{h}_{n i}\right|^{2}\right\}}_{\Xi_{2}},
\end{aligned}
$$

where $(f)$ follows from removing the cross-terms that are zero due to the independence and zero mean of the channel between UE and different APs. Next, we focus on the calculation when the normalized MR combining is utilized at APs, i.e., the calculation of $\Xi_{1}$, which can be further calculated as

$$
\Xi_{1} \stackrel{(g)}{=} \sum_{n \in \mathcal{M}_{k}, n \notin \mathcal{G}} \mathbb{E}\left\{\left|\mathbf{v}_{n k}^{H} \mathbf{D}_{n k} \mathbf{h}_{n i}\right|^{2}\right\}
$$

where $(g)$ follows from the fact that all cross-terms are zero similarly. Note that the calculation of the expectation in (39) depends on whether or not the UE uses the same pilot, i.e., $i \in \mathcal{P}_{k}$ or $i \notin \mathcal{P}_{k}$. When $i \in \mathcal{P}_{k}$, the expectation is given by

$$
\begin{aligned}
& \mathbb{E}\left\{\left|\mathbf{v}_{n k}^{H} \mathbf{D}_{n k} \mathbf{h}_{n i}\right|^{2}\right\}=\mathbb{E}\left\{\left|\frac{\hat{\mathbf{h}}_{n k}^{H}}{\left\|\hat{\mathbf{h}}_{n k}\right\|} \mathbf{D}_{n k}\left(\hat{\mathbf{h}}_{n i}+\tilde{\mathbf{h}}_{n i}\right)\right|^{2}\right\} \\
& =\mathbb{E}\left\{\frac{\hat{\mathbf{h}}_{n k}^{H}}{\left\|\hat{\mathbf{h}}_{n k}\right\|} \mathbf{D}_{n k}\left(\hat{\mathbf{h}}_{n i}+\tilde{\mathbf{h}}_{n i}\right)\left(\hat{\mathbf{h}}_{n i}+\tilde{\mathbf{h}}_{n i}\right)^{H} \mathbf{D}_{n k}^{H} \frac{\hat{\mathbf{h}}_{n k}}{\left\|\hat{\mathbf{h}}_{n k}\right\|}\right\} \\
& \underbrace{\stackrel{(h)}{\approx} \underbrace{\mathbb{E}\left\{\left\|\hat{\mathbf{h}}_{n k}\right\|^{2}\right\}}_{\Theta_{2}\left\{\hat{\mathbf{h}}_{n k}^{H} \mathbf{D}_{n k} \hat{\mathbf{h}}_{n i} \hat{\mathbf{h}}_{n i}^{H} \mathbf{D}_{n k}^{H} \hat{\mathbf{h}}_{n k}\right\}}+\underbrace{\mathbb{E}\left\{\hat{\mathbf{h}}_{n k}^{H} \mathbf{D}_{n k} \tilde{\mathbf{h}}_{n i} \tilde{\mathbf{h}}_{n i}^{H} \mathbf{D}_{n k}^{H} \hat{\mathbf{h}}_{n k}\right\}}_{\mathbb{E}\left\{\left\|\hat{\mathbf{h}}_{n k}\right\|^{2}\right\}}}_{\Theta_{1}}
\end{aligned}
$$

where $(h)$ also follows from the fact that two cross-terms are zeros due to the independence of the channel estimate and estimate error and exploits the first order Taylor approximations to approximate these expectations of quotients. Then, following the same approach as in [25, Corollary. 4.5], we have

$$
\begin{aligned}
\Theta_{1} & =\frac{P_{i} \tau_{p}\left|\operatorname{tr}\left(\mathbf{D}_{n k} \mathbf{R}_{n i} \boldsymbol{\Phi}_{n k} \mathbf{R}_{n k}\right)\right|^{2}}{\operatorname{tr}\left(\mathbf{R}_{n k} \boldsymbol{\Phi}_{n k} \mathbf{R}_{n k}\right)} \\
& +\frac{\operatorname{tr}\left(\mathbf{D}_{n k}\left(\mathbf{R}_{n i}-\mathbf{C}_{n i}\right) \mathbf{R}_{n k} \boldsymbol{\Phi}_{n k} \mathbf{R}_{n k}\right)}{\operatorname{tr}\left(\mathbf{R}_{n k} \boldsymbol{\Phi}_{n k} \mathbf{R}_{n k}\right)} \\
\Theta_{2} & =\frac{\operatorname{tr}\left(\mathbf{D}_{n k} \mathbf{C}_{n i} \mathbf{R}_{n k} \boldsymbol{\Phi}_{n k} \mathbf{R}_{n k}\right)}{\operatorname{tr}\left(\mathbf{R}_{n k} \boldsymbol{\Phi}_{n k} \mathbf{R}_{n k}\right)}
\end{aligned}
$$


When $i \notin \mathcal{P}_{k}$, with the help of [25, Corollary. 4.5], we have

$$
\begin{aligned}
& \mathbb{E}\left\{\left|\mathbf{v}_{n k}^{H} \mathbf{D}_{n k} \mathbf{h}_{n i}\right|^{2}\right\}=\mathbb{E}\left\{\left|\frac{\hat{\mathbf{h}}_{n k}^{H}}{\left\|\hat{\mathbf{h}}_{n k}\right\|} \mathbf{D}_{n k} \mathbf{h}_{n i}\right|^{2}\right\} \\
& =\mathbb{E}\left\{\frac{\hat{\mathbf{h}}_{n k}^{H} \mathbf{D}_{n k} \mathbf{h}_{n i}}{\left\|\hat{\mathbf{h}}_{n k}\right\|} \cdot \frac{\left.\mathbf{h}_{n i}^{H} \mathbf{D}_{n k}^{H} \hat{\mathbf{h}}_{n k}\right\}}{\left\|\hat{\mathbf{h}}_{n k}\right\|}\right\} \stackrel{{ }_{(i)}}{\approx} \frac{\mathbb{E}\left\{\hat{\mathbf{h}}_{n k}^{H} \mathbf{D}_{n k} \mathbf{h}_{n i} \mathbf{h}_{n i}^{H} \mathbf{D}_{n k}^{H} \hat{\mathbf{h}}_{n k}\right\}}{\mathbb{E}\left\{\left\|\hat{\mathbf{h}}_{n k}\right\|^{2}\right\}} \\
& =\frac{\operatorname{tr}\left(\mathbf{D}_{n k} \mathbb{E}\left\{\mathbf{h}_{n i} \mathbf{h}_{n i}^{H}\right\} \mathbb{E}\left\{\hat{\mathbf{h}}_{n k} \hat{\mathbf{h}}_{n k}^{H}\right\}\right)}{\mathbb{E}\left\{\left\|\hat{\mathbf{h}}_{n k}\right\|^{2}\right\}}=\frac{\operatorname{tr}\left(\mathbf{D}_{n k} \mathbf{R}_{n i} \mathbf{R}_{n k} \mathbf{\Phi}_{n k} \mathbf{R}_{n k}\right)}{\operatorname{tr}\left(\mathbf{R}_{n k} \boldsymbol{\Phi}_{n k} \mathbf{R}_{n k}\right)},
\end{aligned}
$$

where $(i)$ also uses the first order Taylor approximation. To the end, the desired results in Corollary can be easily derived.

\section{Acknowledgements}

Not applicable.

Funding

This work was supported in part by the National Natural Science Foundation of China under Grant 62071485, Grant 61901519 and Grant 61771486, and in part by the Basic Research Project of Jiangsu Province under Grant BK20192002 and the Natural Science Foundation of Jiangsu Province under Grant BK20201334, BK20181335.

\section{Abbreviations}

MIMO: Massive multiple-input multiple-output; AP: Access point; IC-IB: Inter-cluster interference-based; SE: Spectral efficiency; UE: User equipment; LP-MMSE: Local partial minimum mean-squared error; MR: Maximum ratio; MMF: Max-min fairness; CPU: Central processing unit; DCC: Dynamic cooperation cluster; RZF: Regularized zero-forcing; SLNR: Signal-to-leakage-and-noise ratio; GPS: Global positioning system; LOS: Line-of-sight; HSR: High-speed railway; LBG: Location-based greedy; ULA: Uniform linear array; AOA: Angle of arrival; MMSE:

Minimum mean-square-error; NMR: Normalized MR; NCB: normalized conjugate beamforming; MSE: Mean-squared error; BPA: Basic pilot assignment.

\section{Availability of data and materials}

Not applicable

\section{Competing interests}

The authors declare that they have no competing interests.

\section{Authors' contributions}

All authors made contributions in the discussions, analyses, and implementation of the proposed solution. CW contributed in writing the manuscript. All authors read and approved the final manuscript.

\section{Author details}

College of Communications Engineering, Army Engineering University of PLA, Nanjing, China.

References

1. H.Q.Ngo, A.Ashikhmin, H.Yang, E.G.Larsson, T.L.Marzetta: Cell-free massive MIMO versus small cells. IEEE Trans. Wireless Commun. 16(3), 1834-1850 (2017)

2. G.Interdonato, E.Björnson, H.Q.Ngo, Pål.Frenger, E.G.Larsson: Ubiquitous cell-free massive MIMO communications. EURASIP J. Wireless Commun. Netw. 2019(197), 1-13 (2019)

3. J.Zhang, E.Björnson, M.Matthaiou, D.W.K.Ng, H.Yang, D.J.Love: Prospective multiple antenna technologies for beyond 5G. IEEE J. Sel. Areas Commun. 38(8), 1637-1660 (2020)

4. Ö.Özdogan, E.Björnson, J.Zhang: Performance of cell-free massive MIMO with rician fading and phase shifts. IEEE Trans. Wireless Commun. 18(11), 5299-5315 (2019)

5. M.Bashar, K.Cumanan, A.G.Burr, M.Debbah, H.Q.Ngo: On the uplink max-min SINR of cell-free massive MIMO systems. IEEE Trans. Wireless Commun. 18(4), 2021-2036 (2019)

6. P.Liu, Luo, K., D.Chen, T.Jiang: Spectral efficiency analysis of cell-free massive MIMO systems with zero-forcing detector. IEEE Trans. Wireless Commun. 19(2), 795-807 (2020)

7. E.Björnson, L.Sanguinetti: Scalable cell-free massive MIMO systems. IEEE Trans. Commun. 68(7), 4247-4261 (2020)

8. S.Buzzi, C.DAndrea: Cell-free massive MIMO: User-centric approach. IEEE Wireless Commun. Lett. 6(6), 706-709 (2017)

9. S.Buzzi, C.DAndrea, A.Zappone, C.DElia: User-centric 5 G cellular networks: Resource allocation and comparison with the cell-free massive MIMO approach. IEEE Trans. Wireless Commun. 19(2), 1250-1264 (2020) 
10. M.Alonzo, S.Buzzi, A.Zappone, C.DElia: Energy-efficient power control in cell-free and user-centric massive MIMO at millimeter wave. IEEE Trans. Green Commun. Netw. 3(3), 651-663 (2019)

11. Ö.T.Demir, E.Björnson, L.Sanguinetti: Foundations of user-centric cell-free massive MIMO. Foundations and Trends ${ }^{\circledR}$ in Signal Processing 14(3-4), 162-472 (2021)

12. E.Björnson, L.Sanguinetti: Making cell-free massive MIMO competitive with MMSE processing and centralized implementation. IEEE Trans. Wireless Commun. 19(1), 77-90 (2020)

13. E.Björnson, L.Sanguinetti: A new look at cell-free massive MIMO: Making it practical with dynamic cooperation. In: Proc. IEEE Annu. Int. Symp. Pers., Indoor, Mobile Radio Commun. (PIMRC), pp. 1-6 (2019)

14. R.Taranto, S.Muppirisetty, R.Raulefs, D.Slock, T.Svensson, H.Wymeersch: Location-aware communications for 5G networks: How location information can improve scalability, latency, and robustness of 5G. IEEE Signal Process. Mag. 31(6), 102-112 (2014)

15. Y.Shen, C.Jiang, T.Quek, Y.Ren: Location-aware device communication design: Exploration and exploitation on energy. IEEE Wireless Commun. 23(2), 46-52 (2016)

16. N.Akbar, S.Yan, N.Yang, J.Yuan: Location-aware pilot allocation in multicell multiuser massive MIMO networks. IEEE Trans. Veh. Technol. 67(8), 7774-7778 (2018)

17. L.S.Muppirisetty, T.Charalambous, J.Karout, G.Fodor, H.Wymeersch: Location-aided pilot contamination avoidance for massive MIMO systems. IEEE Trans. Wireless Commun. 17(4), 2662-2674 (2018)

18. Y.Xu, X.Xia, K.Xu, Y.Wang: Three-dimension massive MIMO for air-to-ground transmission: Location-assisted precoding and impact of AoD uncertainty. IEEE Access 5, 15582-15596 (2017)

19. K.Xu, Z.Shen, Y.Wang, X.Xia: Location-aided mMIMO channel tracking and hybrid beamforming for high-speed railway communications: An angle-domain approach. IEEE Syst. J. 14(1), 93-104 (2020)

20. M.Attarifar, A.Abbasfar, A.Lozano: Random vs structured pilot assignment in cell-free massive MIMO wireless networks. In: Proc. IEEE Int. Conf. Commun., pp. 1-6 (2018)

21. Y.Zhang, H.Cao, P.Zhong, Qi, C., L.Yang: Location-based greedy pilot assignment for cell-free massive MIMO systems. In: Proc. IEEE 4th Int. Conf. Comput. Commun., pp. 392-396 (2018)

22. A.Abdallah, M.M.Mansour: Efficient angle-domain processing for FDD-based cell-free massive MIMO systems. IEEE Trans. Commun. 68(4), 2188-2203 (2020)

23. J.Qiu, K.Xu, Z.Shen: Cooperative fingerprint positioning for cell-free massive MIMO systems. In: Proc. IEEE Int. Conf. Wireless Commun. Signal Process., pp. 1-6 (2020)

24. H.Q.Ngo, L.Tran, T.Q.Duong, M.Matthaiou, E.G.Larsson: On the total energy efficiency of cell-free massive MIMO. IEEE Trans. Green Commun. Netw. 2(1), 25-39 (2018)

25. E.Björnson, J.Hoydis, L.Sanguinetti: Massive MIMO networks: Spectral, energy, and hardware efficiency. Foundations and Trends ${ }^{\circledR}$ in Signal Processing 11(3-4), 154-655 (2017)

26. M.Bashar, K.Cumanan, A.G.Burr, H.Q.Ngo, L.Hanzo, P.Xiao: On the performance of cell-free massive MIMO relying on adaptive NOMA/OMA mode-switching. IEEE Trans. Commun. 68(2), 792-810 (2020)

27. X.Zheng, A.Liu, V.Lau: Joint channel and location estimation of massive MIMO system with phase noise. IEEE Trans. Signal Process. 68, 2598-2612 (2020)

28. S.Chen, J.Zhang, E.Björnson, J.Zhang, B.Ai: Structured massive access for scalable cell-free massive MIMO systems. IEEE J. Sel. Areas Commun. 39(4), 1086-1100 (2021)

29. G.Femenias, F.Riera-Palou, A.Álvarez-Polegre, A.García-Armada: Short-term power constrained cell-free massive-MIMO over spatially correlated ricean fading. IEEE Trans. Veh. Technol. 69(12), 15200-15215 (2020)

30. M.Alageli, A.Ikhlef, F.Alsifiany, M.Abdullah, G.Chen, J.Chambers: Optimal downlink transmission for cell-free SWIPT massive MIMO systems with active eavesdropping. IEEE Trans. Inf. Forensics Security 15, 1983-1998 (2020) 
Table 3 Figure legends.

\begin{tabular}{|c|c|}
\hline Figure number & Title \\
\hline Figure 1 & System model \\
\hline Figure 2(a) & The system model after AP selection \\
\hline Figure 2(b) & Illustration of step 2 for the IC-IB pilot assignment scheme \\
\hline Figure 2(c) & Illustration of step 3 for the IC-IB pilot assignment scheme \\
\hline Figure 3 & MSE of channel estimation with various SNR for different pilot assignment schemes \\
\hline Figure $4(a)$ & The CDF of per-UE uplink SE with different $M$ for different combining schemes \\
\hline Figure 4(b) & The $90 \%$-likely SE with different $M$ for different combining schemes \\
\hline Figure $4(c)$ & The average SE with different $M$ for different combining schemes \\
\hline Figure $4(d)$ & The UE fairness with different $M$ for different combining schemes \\
\hline Figure 5(a) & The CDF of per-UE uplink SE with different thresholds $\gamma$ for different combining schemes \\
\hline Figure $5(\mathrm{~b})$ & The $90 \%$-likely SE with different thresholds $\gamma$ for different combining schemes \\
\hline Figure 5(c) & The average SE with different thresholds $\gamma$ for different combining schemes \\
\hline Figure $5(d)$ & The UE fairness with different thresholds $\gamma$ for different combining schemes \\
\hline Figure $6(a)$ & Uplink sum SE versus $M$ for different combining schemes \\
\hline Figure $6(\mathrm{~b})$ & Uplink sum SE versus $N$ for different combining schemes \\
\hline Figure $7(a)$ & The number of complex multiplications versus $M$ for different combining schemes \\
\hline Figure $7(b)$ & Average SE and the number of complex multiplications versus $\gamma$ for different combining schemes \\
\hline Figure $8(a)$ & The CDF of uplink SE with different power control schemes for different combining schemes \\
\hline Figure 8(b) & The $90 \%$-likely SE with MMF power control for different combining schemes \\
\hline Figure $8(c)$ & The average SE with MMF power control for different combining schemes \\
\hline Figure $8(d)$ & The UE fairness with MMF power control for different combining schemes \\
\hline Figure 8(e) & The $90 \%$-likely SE with full power for different combining schemes \\
\hline Figure 8(f) & The average SE with full power for different combining schemes \\
\hline Figure $8(\mathrm{~g})$ & The UE fairness with full power for different combining schemes \\
\hline
\end{tabular}

